OUTP-97-09P

hep-th/9703071

January 1, 2018

\title{
Liouville Dressed Weights and Renormalization of Spin in Topologically Massive Gravity
}

\author{
Ian I. Kogan and Richard J. Szabo \\ Department of Physics - Theoretical Physics \\ University of Oxford \\ 1 Keble Road, Oxford OX1 3NP, U.K.
}

\begin{abstract}
We examine the relations between observables in two- and three-dimensional quantum gravity by studying the coupling of topologically massive gravity to matter fields in non-trivial representations of the three-dimensional Lorentz group. We show that the gravitational renormalization of spin up to one-loop order in these theories reproduces the leading orders of the KPZ scaling relations for quantum Liouville theory. We demonstrate that the two-dimensional scaling dimensions can be computed from tree-level Aharonov-Bohm scattering amplitudes between the charged particles in the limit where the three-dimensional theory possesses local conformal invariance. We show how the three-dimensional description defines scale-dependent weights by computing the one-loop order anomalous magnetic moment of fermions in a background electromagnetic field due to the renormalization by topologically massive gravity. We also discuss some aspects concerning the different phases of three-dimensional quantum gravity and argue that the topological ones may be related to the branched polymer phase of twodimensional quantum gravity.
\end{abstract}




\section{Introduction}

It has been known for quite some time that gauge and gravity theories in a three-dimensional spacetime can have dynamics which are not possible in other dimensions. Topologically massive gravity [1] is the modification of three-dimensional Einstein gravity by the addition of the gravitational Chern-Simons term to the usual Einstein-Hilbert action. In contrast to the ordinary Einstein theory, which is non-dynamical due to the equivalence of the Einstein and Riemann tensors in three dimensions, it has a propagating, massive graviton degree of freedom. It has been shown [2, 3] to be the unique locally dynamical and unitary gravity model which is power-counting renormalizable, and it exhibits a variety of novel effects such as a gravitational analog of the Aharonov-Bohm effect [4, 5], violation of the equivalence principle, and anti-gravity [4]. In this Paper we will discuss the relationship between topologically massive gravity and the gravity sector of string theory (Liouville theory).

The relation between topologically massive gravity on a three-dimensional spacetime manifold $\mathcal{M}$ with boundary $\partial \mathcal{M}$ and two-dimensional gravity on $\partial \mathcal{M}$ was first conjectured in [6], and then derived by Carlip in [7] from a formal path-integral approach. The induced Liouville gravity theory on $\partial \mathcal{M}$ is [0, 8$]$

$$
S_{L}[\phi]=\int_{\partial \mathcal{M}} d^{2} z\left(k^{\prime} \partial_{\bar{z}} \phi \partial_{z} \phi-Q R^{(2)} \phi+\Lambda \mathrm{e}^{\alpha_{+} \phi}\right)
$$

where $\phi$ is the induced dilaton field, the two-dimensional cosmological constant $\Lambda$ is equal to the square of the three-dimensional topological graviton mass [ $\mathbb{8}$, and $k^{\prime}$ is the gravitational Chern-Simons coefficient which is related to the central charge $c$ of the $S L(2, \mathbb{R})$ Kac-Moody algebra by $c=-k^{\prime}-4$. This connection expands the general relationship between threedimensional topological field theory and two-dimensional conformal field theory [9], and it describes gravitational dressing effects in the topological membrane formulation of string

theory in which the string world-sheet is filled in and viewed as the boundary of a 3-manifold [10, 11].

In the general relationship between topological gauge theories and conformal field theories, one can describe the fundamental quantum observables of the primary conformal fields by coupling the gauge theory to charged matter. The $n$-point correlation functions of the conformal field theory can be decomposed

$$
\left\langle\prod_{i=1}^{n} V\left(z_{i}, \bar{z}_{i}\right)\right\rangle=\left\langle\prod_{i=1}^{n} V_{\mathrm{L}}\left(z_{i}\right)\right\rangle\left\langle\prod_{i=1}^{n} V_{\mathrm{R}}\left(\bar{z}_{i}\right)\right\rangle
$$

in terms of products of left and right conformal blocks, where $V_{\mathrm{L}}\left(z_{i}\right)$ and $V_{\mathrm{R}}\left(\bar{z}_{i}\right)$ are the holomorphic and anti-holomorphic chiral vertex operators corresponding to the left-right symmetric vertex operator $V\left(z_{i}, \bar{z}_{i}\right)$. In the corresponding three-dimensional gauge theory we consider the 3-manifold $\mathcal{M}=\Sigma \times[0,1]$ whose two boundaries $\Sigma_{\mathrm{L}}$ and $\Sigma_{\mathrm{R}}$ are connected by a finite 
interval. A Chern-Simons gauge theory in $\mathcal{M}$ induces both left- and right-moving sectors of the two-dimensional conformal field theory, and an insertion of a vertex operator on the Riemann surface $\Sigma$ is equivalent to insertions of the chiral vertex operators $V_{\mathrm{L}}(z)$ and $V_{\mathrm{R}}(\bar{z})$ on the left- and right-moving worldsheets $\Sigma_{\mathrm{L}}$ and $\Sigma_{\mathrm{R}}$, respectively. The insertions corresponding to the correlation functions (1.2) are induced by path-ordered products of open Wilson line operators $9-12$

$$
W_{\mathcal{C}_{z_{1}, \bar{z}_{1}} ; \ldots ; \mathcal{C}_{z_{n}, \bar{z}_{n}}}^{\left(R_{1}, \ldots, R_{n}\right)}\left[A^{(1)}, \ldots, A^{(n)}\right]=\prod_{i=1}^{n} \operatorname{tr}_{R_{i}} P \exp \left(i \int_{\mathcal{C}_{z_{i}, \bar{z}_{i}}} A^{(i)}\right)
$$

along the oriented paths $\mathcal{C}_{z_{i}, \bar{z}_{i}} \subset \mathcal{M}$ with endpoints $z_{i} \in \Sigma_{\mathrm{L}}$ and $\bar{z}_{i} \in \Sigma_{\mathrm{R}}$. Correlators of insertions of the Wilson lines (1.3) in $\mathcal{M}$ induce phase factors from adiabatical rotation of charged particles coupled to the gauge fields $A^{(i)}$ in the representations $R_{i}$ of a gauge group $G$ (this set of quantum numbers depends on the types of vertex operators under consideration). The quantum particles propagate along $\mathcal{C}_{z_{i}, \bar{z}_{i}}$ from left- to right-moving worldsheets, so that the corresponding linking of the Wilson lines from the adiabatical rotations in $\mathcal{M}$ are equivalent to braidings of the associated vertex operators on $\Sigma$ whose induced phases correspond to the conformal dimensions of the primary fields.

In the connection between three-dimensional gravity and Liouville theory, this approach can therefore provide geometrical and dynamical descriptions of the characteristics of twodimensional quantum gravity within the more conventional framework of quantum field theory. This feature has been exploited recently in [12] where it was demonstrated that the anomalous scaling dimensions of primary operators in the induced conformal field theories can be calculated perturbatively in the three-dimensional quantum field theory by viewing the induced spin as the Aharonov-Bohm phase factor [13 that arises in the scattering amplitudes between dynamical charged particles interacting via exchange of the topologically massive bosons. In topologically massive gravity, the Liouville-dressing of vertex operators on the worldsheet can be expected to coincide with graviton exchanges between charged particles in the bulk. It was argued in [12] that this identification reproduces the leading orders of the Knizhnik-Polyakov-Zamolodchikov (KPZ) scaling relations [14]

$$
\Delta-\Delta_{0}=\frac{\Delta(1-\Delta)}{c+2}
$$

for quantum Liouville theory. The formula (1.4) describes the transformation of the bare spin $\Delta_{0}$ of some primary conformal fields to the spin $\Delta$ due to the dressing by two-dimensional quantum gravity. The iterative large- $k^{\prime}$ expansion of (1.4) for the branch which has $\Delta\left(\Delta_{0}=\right.$ $0)=0$ is

$$
\Delta_{-}=\Delta_{0}+\frac{\Delta_{0}-\Delta_{0}^{2}}{k^{\prime}}+\frac{2 \Delta_{0}^{3}-\Delta_{0}-\Delta_{0}^{2}}{k^{\prime 2}}+\ldots
$$

It was shown in 12 that when three-dimensional charged scalar matter fields, with an anomalous fractional spin $\Delta_{0}$ from their coupling to a Chern-Simons gauge field, are coupled to 
topologically massive gravity, the one-loop order scattering amplitudes reproduce the leading orders of (1.5). This establishes a non-trivial direct link between the observables of the two- and three-dimensional gravity theories. The three-dimensional description of non-critical string theory in this way suggests a clearer, geometrical picture of its observables from a dynamical point of view, and it also provides evidence that a suitably defined quantum measure does indeed exist for the path-integral of the Liouville gravity theory.

In this Paper we shall explore further the relationship between three-dimensional gravitational scattering amplitudes and the gravitational dressing of conformal weights as predicted by the KPZ formula (1.5), emphasizing the roles played by the various symmetry groups of the three-dimensional theory. We will study the coupling of topologically massive gravity to matter fields which carry a non-trivial representation of the local Lorentz group of the three-dimensional spacetime, wherein one expects to be able to analyse tree-level amplitudes because of the non-zero bare spin that the fields possess. We shall see that the tree-level renormalization of spin is controlled by the interactions with the spin-connection field of topologically massive gravity. This analysis illustrates how the various fields of topologically massive gravity conspire in general to dress the bare observables in such a way so as to reproduce the KPZ formula (1.4). In this way we obtain a dynamical picture for the geometric realization of the mysterious "hidden" $S L(2, \mathbb{R}) \cong S O(2,1)$ Kac-Moody symmetry group of Liouville theory [14] as the local Lorentz group of three-dimensional gravity. As we shall see, the interpretation of these dressed spins as Aharonov-Bohm phases requires tuning the topological graviton mass to that point where the theory has local three-dimensional conformal invariance and defines a topological $S O(2,1)$ Chern-Simons gauge theory.

In light of this requirement, we consider also the anomalous spin through the gravitational renormalization of the magnetic moment of fermion fields coupled to a background electromagnetic field. We show that, at one-loop order, this yields an unambiguous definition of the Liouville dressed spins which also agrees with the two-dimensional theory. It does, however, require taking the limit where the topologically massive gravity model reduces to ordinary Einstein gravity (the same limit that was required in [12]), i.e. tuning the topological graviton mass to the point where the theory becomes a topological $I S O(2,1)$ Chern-Simons gauge theory. We will see that the determination of the weights at this $\operatorname{ISO}(2,1)$-invariant point is equivalent to an identification of this limit of topologically massive gravity with three-dimensional topological gravity. This then defines the dressed spins (1.5) in the threedimensional theory for all scales. We shall see in fact that the three-dimensional approach, in contrast to the Liouville theory, defines a scale-dependent conformal dimension through such higher-loop order renormalizations of the spin of the gravity-coupled fermion fields. These results may also shed light on how the ghost sector of Liouville theory is realized in terms of the $S O(2,1)$ spin-connection in topologically massive gravity [15]. 
The organization of this Paper is as follows. In Section 2 we review the first-order formalism for topologically massive gravity and write down its Feynman rules. In Section 3 we briefly review the perturbative approach to determining conformal dimensions and argue heuristically how this will reproduce the leading orders of the KPZ formula when the gravity theory is coupled to spinning matter fields. In Section 4 we verify these arguments by explicit tree-level calculations for a few cases and point out some intriguing features of the amplitudes. In Section 5 we compute the one-loop order renormalization of the fermionic magnetic moment and discuss the scale dependence of the weights that are so derived. Finally, in Section 6 we discuss the possibility of connecting different phases of topologically massive gravity, including the one that admits a spacetime with a conical singularity (and hence a gravitational Aharonov-Bohm effect), with models of two-dimensional quantum gravity coupled to $c>1$ conformal matter fields. In these cases the perturbative approach breaks down, so that the apparent mystery of the two-dimensional theories in these instances are evident in the three-dimensional gravity models as well. An Appendix at the end of the Paper summarizes some of the identities used for the more complicated calculations.

\section{Topologically Massive Gravity in the First Order For- malism}

We begin by briefly reviewing the basic properties of topologically massive gravity and its Feynman rules. We will be interested in describing this model within the first order formalism for general relativity [16]. For this, we introduce the dreibein fields $e^{a}=e_{\mu}^{a} d x^{\mu}$ which span the frame bundle of the oriented three-dimensional spacetime manifold $\mathcal{M}$ which has metric of Minkowski signature. Here and in the following greek letters will label the spacetime indices (i.e. the components of the local basis vectors of the tangent space) and latin letters will denote the basis indices of the local Lorentz group $S O(2,1)$ of the tangent bundle. The dreibein fields are related to the metric $g$ of $\mathcal{M}$ by the orthonormality condition $g^{\mu \nu} e_{\mu}^{a} e_{\nu}^{b}=$ $\eta^{a b}=\operatorname{diag}(1,-1,-1)$, or equivalently by the completeness relation

$$
\eta_{a b} e^{a} \otimes e^{b}=g
$$

The action for topologically massive gravity in the first order formalism is

$$
S_{T M G}=S_{E}+S_{C S}^{(\text {grav })}+S_{\lambda}
$$

where

$$
S_{C S}^{\text {(grav) }}=\frac{k^{\prime}}{8 \pi} \int_{\mathcal{M}}\left(\omega^{a} \wedge d \omega^{a}+\frac{2}{3} \epsilon^{a b c} \omega^{a} \wedge \omega^{b} \wedge \omega^{c}\right)
$$


is the parity-odd gravitational Chern-Simons action, and $\omega^{a}=\epsilon^{a b c} \omega^{b c}$ with $\omega^{a b}=-\omega^{b a}=$ $\omega_{\mu}^{a b} d x^{\mu}$ the spin-connection of the frame bundle of $\mathcal{M}$. We use the convention $\epsilon^{012}=+1$ for the antisymmetric tensor, and we shall always assume that the gravitational Chern-Simons coefficient $k^{\prime} \in \mathbb{R}$ is large enough so that perturbation theory makes sense. The EinsteinHilbert action is

$$
S_{E}=\kappa \int_{\mathcal{M}} e^{a} \wedge R^{a}
$$

where

$$
R^{a}=R_{\mu \nu}^{a} d x^{\mu} \wedge d x^{\nu}=d \omega^{a}+\epsilon^{a b c} \omega^{b} \wedge \omega^{c}
$$

is the curvature of the spin-connection $\omega^{a}$, and $\kappa$ is the Planck mass. We have also included in (2.2) the term

$$
S_{\lambda}=\int_{\mathcal{M}} \lambda^{a} \wedge\left(d e^{a}+2 \epsilon^{a b c} \omega^{b} \wedge e^{c}\right)
$$

where $\lambda^{a}=\lambda_{\mu}^{a} d x^{\mu}$ are Lagrange multiplier fields that enforce the constraint which ensures that the usual covariant derivative $\nabla$ constructed from the spin-connection is compatible with the metric $g$ (i.e. $\nabla e^{a}=0$ ) so that the Einstein-Hilbert action in the first order formalism coincides with the usual one of general relativity. This means that we are working in the minimal formalism for general relativity in which $\omega$ is the Levi-Civita connection of the spin bundle of $\mathcal{M}$.

The ordinary, pure Einstein theory (2.4) in three-dimensions has no propagating degrees of freedom and is a topological field theory. In fact, it can be regarded as a topological ChernSimons gauge theory with gauge group defined by the Poincaré group $I S O(2,1) \supset S O(2,1)$ of the spacetime [17]. The addition of the gravitational Chern-Simons term (2.3) makes the gravitons of the theory massive with topological mass

$$
M=8 \pi \kappa / k^{\prime}
$$

In the infrared limit $M \rightarrow \infty$ (equivalently $k^{\prime} \rightarrow 0$ ) this propagating degree of freedom decouples and the Chern-Simons term in $(2.2)$ becomes irrelevant. Then the kinetic term in the induced Liouville action (1.1) vanishes and the gravity theory (2.2) induces the topological field theory on $\partial \mathcal{M}$ which describes the moduli space of Riemann surfaces. This was first argued in [17] where it was observed that the physical phase space for three-dimensional Einstein gravity is closely related to the moduli space of complex structures. It was subsequently shown in [11] that the only contribution to the gauge-fixed path integral over three-dimensional metrics in the interior of $\mathcal{M}$ is from an integration over the moduli space of $\partial \mathcal{M}$ with the same integration measure (the Weil-Petersson measure) that naturally arises in string theory. The resulting quantum mechanics on moduli space was also studied in [11]. For finite $M$, the world-sheet and bulk scales are related by

$$
\Lambda=M^{2}
$$


which establishes a non-trivial correspondence between the quantum Liouville theory and topologically massive gravity. Some values of the world-sheet cosmological constant $\Lambda$ then correspond to different symmetries of the three-dimensional theory. Various physical applications of this feature, for example to $S L(2, \mathbb{R}) / U(1)$ black holes and $c=1$ strings, are discussed in [8], and the quantum mechanics on moduli spaces in the full topologically massive gravity theory is studied in [6].

The inclusion of the gravitational Chern-Simons term regulates the ultraviolet divergences of the pure Einstein gravitational field theory. The effective coupling constant of the topologically massive gravity theory is the super-renormalizable, dimensionless expansion parameter $M / \kappa \sim 1 / k^{\prime}$. The topological graviton mass also regulates infrared divergences of the pure Einstein theory. The action (2.2) is diffeomorphism invariant (i.e. generally covariant) and it also possesses a local $S O(2,1)$-invariance defined by rotations of the dreibein fields. The spin-connection is a gauge field of the local Lorentz group, and (2.3) can be regarded as a Chern-Simons action for an $S O(2,1)$ gauge theory with connection $\omega$. Both the dreibein and Lagrange multiplier fields above transform in the adjoint representation of this gauge group. With this point of view, we will study the model (2.2) perturbatively by expanding the graviton field about a flat background metric. This can be done in the "perturbative phase" of the theory wherein $\left\langle e_{\mu}^{a}\right\rangle=\delta_{\mu}^{a}[\mathbb{8}$. Then we shift the dreibein fields as

$$
e_{\mu}^{a} \rightarrow e_{\mu}^{a}+\delta_{\mu}^{a}
$$

and view the topologically massive gravity theory as a quantum field theory on a flat space. Introducing the new variables $\beta^{a}$ defined by

$$
\beta^{a}=\lambda^{a}+\kappa \omega^{a}
$$

the topologically massive gravity action becomes

$$
\begin{gathered}
S_{T M G}=\int d^{3} x\left\{\epsilon^{\mu \nu \lambda} \beta_{\mu}^{a} \partial_{\nu} e_{\lambda}^{a}+2\left(\beta_{\mu}^{\mu} \omega_{\nu}^{\nu}-\beta_{\nu}^{\mu} \omega_{\mu}^{\nu}\right)+\frac{k^{\prime}}{8 \pi} \epsilon^{\mu \nu \lambda} \omega_{\mu}^{a} \partial_{\nu} \omega_{\lambda}^{a}-\kappa\left(\omega_{\mu}^{\mu} \omega_{\nu}^{\nu}-\omega_{\nu}^{\mu} \omega_{\mu}^{\nu}\right)\right. \\
\left.+\epsilon^{\mu \nu \lambda} \epsilon^{a b c}\left(2 \beta_{\mu}^{a} \omega_{\nu}^{b} e_{\lambda}^{c}-\kappa e_{\mu}^{a} \omega_{\nu}^{b} \omega_{\lambda}^{c}+\frac{k^{\prime}}{12 \pi} \omega_{\mu}^{a} \omega_{\nu}^{b} \omega_{\lambda}^{c}\right)\right\}
\end{gathered}
$$

where here and in the following all repeated indices are understood to be summed over by contracting with the flat Minkowski metric $\eta^{a b}$. We shall ignore the gauge-fixing and ghost field terms which are irrelevant for what follows.

The Feynman rules for topologically massive gravity in the first order formalism can now be read off from the action (2.11). In the following we shall work in the transverse Landau gauge. The $e \beta$ and spin-connection propagators are 12

$$
\left\langle\left\langle\beta_{\mu}^{i}(p) e_{\nu}^{j}(-p)\right\rangle=\eta^{i j} \frac{\epsilon_{\mu \nu \lambda} p^{\lambda}}{p^{2}} \quad, \quad\left\langle\left\langle\omega_{\mu}^{i}(p) \omega_{\nu}^{j}(-p)\right\rangle=\frac{4 \pi i}{k^{\prime}} \Omega_{\mu \nu}^{i j}(p)\right.\right.
$$


where the averages $\langle\langle\cdot\rangle\rangle$ denote free field Gaussian correlators in momentum space, and

$$
\begin{aligned}
\Omega_{\mu \nu}^{i j}(p)= & \frac{M\left(\Lambda_{\mu}^{i}(p) \Lambda_{\nu}^{j}(p)-\Lambda_{\mu \nu}(p) \Lambda^{i j}(p)-\Lambda_{\mu}^{j}(p) \Lambda_{\nu}^{i}(p)\right)}{2\left(p^{2}-M^{2}\right)}-\frac{\left(\Lambda_{\mu \nu}(p) \Lambda^{i j}(p)-\Lambda_{\mu}^{j}(p) \Lambda_{\nu}^{i}(p)\right)}{2 M} \\
& -\frac{1}{2 M^{3}}\left(\Lambda_{\mu \nu}(p) p^{i} p^{j}+\Lambda^{i j}(p) p_{\mu} p_{\nu}-\Lambda_{\mu}^{j}(p) p^{i} p_{\nu}-\Lambda_{\nu}^{i}(p) p_{\mu} p^{j}\right) \\
& -\frac{i\left(\epsilon_{\mu \nu \lambda} p^{\lambda} \Lambda^{i j}(p)+\epsilon^{i j k} p_{k} \Lambda_{\mu \nu}(p)\right)}{2\left(p^{2}-M^{2}\right)} \\
& -\frac{i}{2 M^{2}}\left(\epsilon^{i \lambda}{ }_{\mu} p_{\lambda} \frac{p_{\nu} p^{j}}{p^{2}}+\epsilon_{\nu}^{\lambda j} p_{\lambda} \frac{p_{\mu} p^{i}}{p^{2}}+\epsilon_{\mu \lambda \nu} p^{\lambda} \frac{p^{i} p^{j}}{p^{2}}-\epsilon^{i \lambda j} p_{\lambda} \frac{p_{\mu} p_{\nu}}{p^{2}}\right)
\end{aligned}
$$

with

$$
\Lambda_{\mu \nu}(p)=\eta_{\mu \nu}-p_{\mu} p_{\nu} / M^{2}
$$

The pure graviton and we propagators can then be obtained from the momentum space convolutions

$$
\begin{aligned}
D_{\mu \nu}^{i j}(p) & \equiv\left\langle\left\langle e_{\mu}^{i}(p) e_{\nu}^{j}(-p)\right\rangle\right\rangle \\
& =-4\left\langle\left\langle e_{\mu}^{i}(p) \beta_{\lambda}^{k}(-p)\right\rangle\right\rangle\left[\Sigma^{k l}\right]_{\lambda \rho}\left\langle\left\langle\omega_{\rho}^{l}(p) \omega_{\alpha}^{m}(-p)\right\rangle\right\rangle\left[\Sigma^{m n}\right]_{\alpha \sigma}\left\langle\left\langle\beta_{\sigma}^{n}(p) e_{\nu}^{j}(-p)\right\rangle\right\rangle \\
E_{\mu \nu}^{i j}(p) & \equiv\left\langle\left\langle\omega_{\mu}^{i}(p) e_{\nu}^{j}(-p)\right\rangle\right\rangle=2 i\left\langle\left\langle\omega_{\mu}^{i}(p) \omega_{\lambda}^{k}(-p)\right\rangle\right\rangle\left[\Sigma^{k l}\right]_{\lambda \rho}\left\langle\left\langle\beta_{\rho}^{l}(p) e_{\nu}^{j}(-p)\right\rangle\right\rangle
\end{aligned}
$$

where

$$
\left[\Sigma^{k l}\right]_{\lambda \rho}=\delta_{\lambda}^{k} \delta_{\rho}^{l}-\delta_{\rho}^{k} \delta_{\lambda}^{l}
$$

is the spin matrix generator on the space of spin-1 fields. This yields [12]

$$
\begin{aligned}
D_{\mu \nu}^{i j}(p)=\frac{i}{\kappa}( & \frac{M^{2}}{2 p^{2}\left(p^{2}-M^{2}\right)}\left\{\left(\frac{p^{2}}{M^{2}}-2\right) \eta_{\mu \nu}^{\perp}(p) \eta^{\perp i j}(p)+\delta_{\mu}^{\perp i}(p) \delta_{\nu}^{\perp j}(p)+\delta_{\mu}^{\perp j}(p) \delta_{\nu}^{\perp i}(p)\right\} \\
& \left.+\frac{i M}{4} \frac{p^{\lambda}}{p^{2}\left(p^{2}-M^{2}\right)}\left\{\epsilon_{\mu \lambda}^{i} \delta_{\nu}^{\perp j}(p)+\epsilon_{\mu \lambda}{ }^{j} \delta_{\nu}^{\perp i}(p)+\epsilon_{\nu \lambda}{ }^{i}{ }_{\lambda} \delta_{\mu}^{\perp j}(p)+\epsilon_{\nu \lambda}{ }^{j} \delta_{\mu}^{\perp i}(p)\right\}\right) \\
E_{\mu \nu}^{i j}(p)= & -\frac{8 \pi i}{k^{\prime} M}\left(\frac{i \epsilon_{\mu \nu \lambda} p^{\lambda}}{p^{2}} \eta^{i j}-\Omega_{\mu \lambda}^{i j}(p) \delta_{\nu}^{\perp \lambda}(p)\right)
\end{aligned}
$$

where

$$
\eta_{\mu \nu}^{\perp}(p)=\eta_{\mu \nu}-p_{\mu} p_{\nu} / p^{2}
$$

is the symmetric, transverse projection operator on the momentum space of vectors. The Green's function $D_{\mu \nu}^{i j}(p)$ is the usual Deser-Yang graviton propagator [2]. 


\section{Anomalous Spin in Topologically Massive Gravity}

We shall now outline the method, described in detail in [12], for identifying the conformal dimensions of primary operators in two-dimensional conformal field theory as the transmuted spins that appear in charged particle scattering amplitudes in three-dimensional perturbative gauge theory. The minimal coupling of a Chern-Simons gauge field $A=A_{\mu}^{a} T^{a} d x^{\mu}$ to a conserved matter current $J^{\mu}=J_{a}^{\mu} R^{a}$ is described by the parity-violating action

$$
\begin{aligned}
S_{C S}^{[G]}+S_{J} & =\int_{\mathcal{M}} \frac{k}{4 \pi} \operatorname{tr}\left(A \wedge d A+\frac{2}{3} A \wedge A \wedge A\right)+\int_{\mathcal{M}} 2 \operatorname{tr} A \wedge \star J \\
& =\int_{\mathcal{M}} d^{3} x \frac{k}{4 \pi} \epsilon^{\mu \nu \lambda} \operatorname{tr}\left(A_{\mu} \partial_{\nu} A_{\lambda}+\frac{2}{3} A_{\mu} A_{\nu} A_{\lambda}\right)+\int_{\mathcal{M}} d^{3} x \sqrt{g} 2 \operatorname{tr} J^{\mu} A_{\mu}^{a} R^{a}
\end{aligned}
$$

where $R[G]$ is a unitary irreducible representation of the semi-simple gauge group $G$ whose Hermitian generators $T^{a}$ are normalized as $\operatorname{tr} T^{a} T^{b}=-\frac{1}{2} \delta^{a b}$. Again we omit the gauge-fixing and ghost field terms which play no role in the following. The momentum space bare gluon propagator from (3.1) in the transverse Landau gauge is

$$
\mathcal{G}_{\mu \nu}^{a b}(p) \equiv\left\langle\left\langle A_{\mu}^{a}(p) A_{\nu}^{b}(-p)\right\rangle\right\rangle=-\frac{4 \pi}{k} \delta^{a b} \frac{\epsilon_{\mu \nu \lambda} p^{\lambda}}{p^{2}}
$$

The conformal dimension $\Delta$ of a primary operator in the induced conformal field theory on $\partial \mathcal{M}$ can then be determined as the transmuted spin factor that appears in the invariant amplitude for the scattering of two charged particles, of initial momenta $p_{1}$ and $p_{2}$ represented by the current $J$, in the infrared (non-relativistic) regime $q^{2} \rightarrow 0$ of the quantum field theory (Fig. 1),

$$
\mathcal{A}\left(p_{1}, p_{2} ; q\right) \equiv i \operatorname{tr} J^{\mu}\left(2 p_{1}-q\right) \mathcal{G}_{\mu \nu}(q) J^{\nu}\left(2 p_{2}+q\right)=-16 \pi i \operatorname{dim}(G) \Delta(k) \frac{\epsilon_{\mu \nu \lambda} p_{1}^{\mu} p_{2}^{\nu} q^{\lambda}}{q^{2}}
$$

where $q$ is the momentum transfer, and

$$
\Delta(k)=T_{R}[G] \sum_{n \geq 0} \frac{\Delta^{(n)}}{k^{n}}
$$

is the anomalous spin of the charged particles induced by their interaction with the ChernSimons gauge field $A$. It can be computed order by order perturbatively in the Chern-Simons coupling constant $1 / k$. The coefficients $\Delta^{(n)}$ of the expansion (3.4) depend only on invariants of the gauge group $G$ and of the local Lorentz group of the spacetime, and

$$
T_{R}[G] \mathbf{1}=\sum_{a=1}^{\operatorname{dim} G} R^{a} R^{a}
$$

is the quadratic Casimir operator of the Lie group $G$ in the representation $R[G]$. In the (non-relativistic) center of momentum frame, the amplitude (3.3) coincides with the usual 


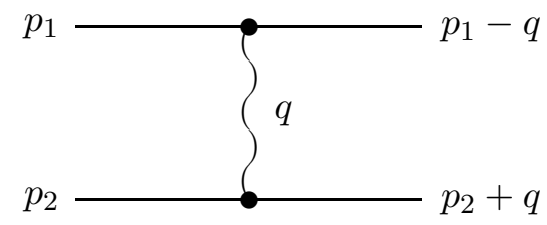

Figure 1: The scattering amplitude for two charged particles. Here $p_{1}, p_{2}$ denote the incoming particle momenta and $q$ is the momentum transfer. Straight lines denote the external charged matter fields, wavy lines depict the gauge fields, and the solid circles represent the minimal coupling of the particle current $J^{\mu}$ to the gluon field.

Aharonov-Bohm amplitude for the scattering of a charge off of a flux [12, 13, 18] which is observable as a long-ranged effect in the theory.

It was demonstrated in [12] that the leading order, tree-level amplitude in the case where $J$ represents charged scalar matter fields yields

$$
\Delta^{(1)}=1
$$

From this result we can naively suggest how the effect of the spin connection in the topologically massive gravity action can reproduce the leading orders of the KPZ formula (1.5). We are interested in coupling the gravity theory of the previous Section to some spinning matter fields. For this, we consider a dynamical $(2 j+1)$-component field $\Phi^{(j)}$ in the unitary irreducible spin- $j$ representation of the local Lorentz group $S O(2,1)$ of the spacetime, where $2 j \in \mathbb{Z}^{+}$. According to (1.5) the bare spin $\Delta_{0}=j$ of these fields should renormalize as

$$
\Delta_{j}\left(k^{\prime}\right)=j-j(j-1) / k^{\prime}+j\left(2 j^{2}-1-j\right) / k^{2}+\ldots
$$

as a result of the gravitational dressing in the induced Liouville gravity theory on $\partial \mathcal{M}$.

As mentioned before, the spin-connection $\omega_{\mu}^{i}$ is a gauge connection of the non-compact Lie group $S O(2,1)$, and so the kinetic terms in the Lorentz invariant action for the minimal coupling of the spin- $j$ fields $\Phi^{(j)}$ to the spin-connection will be constructed from the usual gauge-covariant derivatives

$$
\nabla_{\mu} \Phi^{(j)}=\partial_{\mu} \Phi^{(j)}-i \Sigma_{i}^{(j)} \omega_{\mu}^{i} \Phi^{(j)}
$$

where $\Sigma_{i}^{(j)}$ are the generators of the $(2 j+1)$-dimensional spin- $j$ representation of $S O(2,1)$. To obtain a diffeomorphism invariant action, the covariant derivatives (3.8) will appear contracted with the metric tensor density $\sqrt{g} g^{\mu \nu}$ of the spacetime which will lead to additional interactions with the dreibein fields $e_{\mu}^{i}$. Since the gravitational Chern-Simons term can be regarded as an $S O(2,1)$ gauge field Chern-Simons term, we could naively expect that the 
induced spin from the exchange of one spin-connection coincides with the tree-level gauge theory result in (3.4), (3.6). Then replacing $k$ by $k^{\prime}$ in (3.4) and noting that the quadratic Casimir eigenvalue of the spin- $j$ representation of $S O(2,1)$ is

$$
T_{j}[S O(2,1)]=-j(j-1)
$$

we see that this naive evaluation leads to the order $1 / k^{\prime}$ term in the KPZ formula (3.7). However, this heuristic argument, which connects the basic symmetry group of topologically massive gravity with the hidden $S L(2, \mathbb{R})$ symmetry group of two-dimensional quantum gravity, is not quite precise because the spin-connection is related to the dreibein field by the Cartan-Maurer equation $\nabla e^{a}=0$. For this to be the total tree-level conformal weight requires that the parity odd parts of the one-graviton and we exchange interactions vanish. In the following we shall see that this is indeed the case, i.e. the total tree-level conformal dimension, described as above by a parity-odd Aharonov-Bohm type scattering amplitude, is determined solely by the interaction with the spin-connection $\omega$. This property is, as we will see, a consequence of the dynamical nature of the gravitationally interacting particles [12].

Notice though that this argument breaks down for higher-loop orders. For instance, the one-loop (two-gluon) exchange contribution to the conformal weight in Chern-Simons gauge theory coupled to charged scalar fields is [12

$$
\Delta^{(2)}=-C_{2}[G] \operatorname{sgn}(k)
$$

with $C_{2}[G]$ the dual Coxeter number of the gauge group $G$. The weight (3.10) does not coincide with the $\mathcal{O}\left(1 / k^{2}\right)$ term in the expansion (3.7) using the above naive arguments. We do expect that the amplitudes corresponding to the exchange of two spin connection fields will contribute a term analogous to (3.10) (with $C_{2}[S O(2,1)]=-2$ ), but there will be additional non-vanishing contributions from amplitudes involving a mixing of the spin connection with the other gravity fields. Furthermore, as discussed in [12], in the gravitational case we do not anticipate a dependence on the sign of the Chern-Simons coefficient $k^{\prime}$, as is the case in Chern-Simons gauge theory. The contributions from sole graviton (and other mediating boson) exchanges that do not involve $\omega$ do, however, vanish [12]. The mattercoupled topologically massive gravity theory in this way provides a dynamical illustration of the role of the $S L(2, \mathbb{R}) \cong S O(2,1)$ current algebra symmetry of quantum Liouville theory.

In the following we shall compute tree-level amplitudes in the topologically massive gravity theory coupled to spinning fields. First, we point out two features of the above identification of the conformal dimensions. The first one is that the gravitational renormalization (3.7) produces only one chiral component of the dressed spin. The full spin in the conformal field theory is determined as the difference $\Delta-\bar{\Delta}$ of the weights for the holomorphic and antiholomorphic sectors of the world-sheet theory. The Aharonov-Bohm amplitude therefore only 
describes the holomorphic (or anti-holomorphic) observables in the induced conformal field theory on $\partial \mathcal{M}$, i.e. the spins with $\bar{\Delta}=0$. Thus, strictly speaking, the induced spins that we calculate in this way are really the chiral scaling dimensions. This is evident from the relationship between vertex operators of the induced two-dimensional conformal field theory on $\partial \mathcal{M}$ and Wilson line operators of the three-dimensional gauge theory in $\mathcal{M}$ that was discussed in Section 1.

The second feature concerns the structure of the tree-level amplitude for fermion fields of mass $m$ in a representation $R[G]$ of the gauge group $G$ minimally coupled to a Chern-Simons gauge field. Then the matter field current is $J_{a}^{\mu}=\bar{\psi}_{A} \gamma^{\mu} R_{A B}^{a} \psi_{B}$ (see the next Section), and the scattering amplitude is 18

$$
\mathcal{A}_{f}\left(p_{1}, p_{2} ; q\right)=-\frac{4 \pi \operatorname{dim}(G) T_{R}[G]}{k m}+\frac{4 \pi i \operatorname{dim}(G) T_{R}[G]}{k m^{2}} \frac{\epsilon_{\mu \nu \lambda} p_{1}^{\mu} p_{2}^{\nu} q^{\lambda}}{q^{2}}
$$

in the infrared limit $q^{2} \rightarrow 0$. The first term comes from the finite renormalization of the $U(1)$ charge and corresponds to a short-ranged Pauli magnetic moment interaction arising from the bare spin of the fermion fields. The imaginary, parity-odd second term in (3.11) which has a simple pole at $q^{2}=0$ leads to the usual Aharonov-Bohm interaction and identifies the induced spin as in (3.6). Note that by dimensional analysis the spinor vertices are down by a factor of $2 m$ with respect to those of scalar fields, so that the overall amplitude is suppressed by a factor of $4 m^{2}$ in its relation to the conformal dimensions.

\section{$4 \quad$ KPZ Weights as $S O(2,1)$ Anomalous Spin}

Given the above identification of the KPZ conformal weights as the parity-odd, singular pole terms of the scattering amplitudes, we shall now demonstrate explicitly how this structure appears in topologically massive gravity. The relevant Aharonov-Bohm type contributions can come from the parity odd parts of the gravitational propagators in Section 2, but, as we shall now discuss, there are some subtleties in this description in the pure gravitational case. In this Section we present explicit tree-level calculations to illustrate the discussion above.

\subsection{Scalar Representations}

The simplest case of charged scalar fields $\Phi^{(0)}$ (i.e. the trivial spin $j=0$ representation $\left.\Sigma_{i}^{(0)}=0\right)$ coupled to gravity is described by the action

$$
S_{s}=\int_{\mathcal{M}}\left((\nabla \phi)^{*} \wedge \star \nabla \phi-m^{2} \phi^{*} \star \phi\right)=\int_{\mathcal{M}} d^{3} x \sqrt{g}\left(g^{\mu \nu} \partial_{\mu} \phi^{*} \partial_{\nu} \phi-m^{2} \phi^{*} \phi\right)
$$


where the meson mass $m$ is used to regulate infrared divergences arising from the matter loops. In this case, there is no interaction with the spin-connection and the parity-odd parts of the graviton exchange diagrams vanish to all orders of perturbation theory [12]. To see this, we use the shifts (2.9) to expand the metric determinant factor in (4.1) as

$$
\sqrt{g}=1-\frac{1}{2} h_{\mu}^{\mu}+\frac{1}{4}\left(\frac{1}{2} h_{\mu}^{\mu} h_{\nu}^{\nu}-h_{\nu}^{\mu} h_{\mu}^{\nu}\right)+\ldots
$$

where

$$
g_{\mu \nu}=\eta_{\mu \nu}+e_{\mu}^{a} e_{\nu}^{a}-\eta_{\mu a} e_{\nu}^{a}-\eta_{\nu a} e_{\mu}^{a} \equiv \eta_{\mu \nu}+h_{\mu \nu}
$$

is the expansion of the dynamical metric field about the flat background. Then the mesonmeson-graviton vertex is

$$
\mathcal{E}_{i}^{\mu}\left(p, p^{\prime} ; q\right)=i\left(\delta_{i}^{\mu}\left[p \cdot p^{\prime}-m^{2} / 2\right]+p^{\prime \mu} p_{i}+p^{\mu} p_{i}^{\prime}\right)
$$

where $q=p-p^{\prime}$, and the parity-odd $\epsilon$-part of the tree-level amplitude for the exchange of one graviton between the charged mesons is (Fig. 1 with the straight lines denoting the meson fields and the wavy line representing the dreibein field $e_{\mu}^{a}$ )

$$
\mathcal{B}_{s}\left(p_{1}, p_{2} ; q\right)^{\text {odd }}=i \mathcal{E}_{i}^{\mu}\left(p_{1}, p_{1}-q\right) \mathcal{E}_{j}^{\nu}\left(p_{2}, p_{2}+q\right) D_{\mu \nu}^{i j}(q)^{\text {odd }} \equiv 0
$$

The tree amplitude (4.5) vanishes exactly for all ranges of the momentum transfer $q$. The vanishing at higher-loop orders is then a general consequence of the minimal coupling of the dreibein fields in a transverse gauge to the conserved, dynamical gravitational particle current 12 .

Thus, in the case of the coupling of topologically massive gravity to dynamical charged scalar fields, we can reproduce correctly the KPZ formula (3.7) for a bare $\operatorname{spin} j=0$, i.e.

$$
\Delta_{0}\left(k^{\prime}\right)=0
$$

It was also shown in [12, up to one-loop order, that the gravitational dressing of an anomalous fractional spin $\Delta_{0}=1 / k$ of the scalar fields also leads to the anticipated result. In the following we shall be concerned with this renormalization for higher-spin representations of the local Lorentz group of the spacetime.

\subsection{Spinor Representations}

Next we consider the coupling of topologically massive gravity to fields $\Phi^{(1 / 2)}$ in the lowest non-zero spin $j=1 / 2$ representation of the local Lorentz group. The coupling to spinor fields in the $(2+1)$-dimensional case is especially intriguing because the gamma-matrices obey the identity

$$
\gamma_{\mu} \gamma_{\nu}=g_{\mu \nu}+i \epsilon_{\mu \nu \lambda} \gamma^{\lambda}
$$


and so the Dirac matrices themselves generate a representation of the $S O(2,1)$ Lie algebra, i.e. $\Sigma_{i}^{(1 / 2)}=\gamma_{i} / 2$. The irreducible two-dimensional spinor representation is defined by the Pauli spin matrices

$$
\gamma_{0}=\sigma^{3}=\left(\begin{array}{cc}
1 & 0 \\
0 & -1
\end{array}\right) \quad, \quad \gamma_{1}=i \sigma^{1}=\left(\begin{array}{cc}
0 & i \\
i & 0
\end{array}\right) \quad, \quad \gamma_{2}=i \sigma^{2}=\left(\begin{array}{cc}
0 & 1 \\
-1 & 0
\end{array}\right)
$$

The unique, Lorentz-invariant and lowest order derivative action for Dirac fermion fields is defined by the usual Dirac kinetic term with a minimal coupling to the spin-connection $\omega_{\mu}^{i}$ as described above. Thus we can couple topologically massive gravity to fermion fields by the action

$$
S_{F}=\int_{\mathcal{M}}(\bar{\psi} i \gamma \wedge \star \nabla \psi-m \bar{\psi} \star \psi)=\int_{\mathcal{M}} d^{3} x\left[\sqrt{g} g^{\mu \nu} \bar{\psi} i \gamma_{\mu}\left(\partial_{\nu}-\frac{i}{2} \gamma_{i} \omega_{\nu}^{i}\right) \psi-\sqrt{g} m \bar{\psi} \psi\right]
$$

where $\psi(x)$ are two-component fermion fields and $m$ is the fermion mass which is again introduced to avoid infrared divergence problems. Note that in a three-dimensional spacetime $m$ can be either positive or negative. To find the tree level gravity interactions, we use the metric expansions (4.2) and (4.3). For the tree-level amplitudes, it suffices to keep only terms linear in the dreibein field. Then the $\bar{\psi} \psi \omega$ vertex is

$$
\mathcal{W}_{i}^{\mu}\left(p, p^{\prime} ; q\right)=-\frac{i}{2} \gamma^{\mu} \gamma_{i}=-\frac{i}{2}\left(\delta_{i}^{\mu}+i \epsilon^{\mu}{ }_{i \lambda} \gamma^{\lambda}\right)
$$

where $q=p-p^{\prime}$ and we have used (4.7). The $\bar{\psi} \psi e$ vertex is

$$
\mathcal{G}_{i}^{\mu}\left(p, p^{\prime} ; q\right)=\frac{i}{2}\left[2\left(p+p^{\prime}\right)^{\mu} \gamma_{i}+\delta_{i}^{\mu} \gamma^{\lambda}\left(p+p^{\prime}\right)_{\lambda}+2 m \delta_{i}^{\mu}\right]
$$

with $q=p-p^{\prime}$.

There are four tree-level amplitudes in this gravitationally dressed field theory, which are represented in Fig. 1 with the straight lines denoting the fermion fields and the wavy line depicting an exchange of the spin-connection, graviton, we or ew fields. First, we consider the renormalization due to the spin-connection. The amplitude is

$$
\mathcal{A}_{f}^{\text {(grav) }}\left(p_{1}, p_{2} ; q\right)=\frac{4 \pi i}{k^{\prime}} \bar{u}\left(p_{1}-q\right) \mathcal{W}_{i}^{\mu} u\left(p_{1}\right) \Omega_{\mu \nu}^{i j}(q) \bar{u}\left(p_{2}+q\right) \mathcal{W}_{j}^{\nu} u\left(p_{2}\right)
$$

where $u(p)$ are the on-shell positive energy Dirac spinors. They obey the momentum space Dirac equation

$$
\left(p_{\mu} \gamma^{\mu}-m\right) u(p)=\bar{u}(p)\left(p_{\mu} \gamma^{\mu}-m\right)=0
$$

whose solutions with the Bjorken-Drell normalization $\bar{u}(p) u(p)=1$ are

$$
u(p)=\frac{1}{2 m\left(p^{0}+m\right)}\left(\begin{array}{c}
p^{0}+m \\
-i\left(p^{1}+i p^{2}\right)
\end{array}\right)
$$


with the on-shell condition $(p)^{2}=\left(p^{0}\right)^{2}-\left(p^{1}\right)^{2}-\left(p^{2}\right)^{2}=m^{2}$. Here we have assumed for definiteness that $m>0$.

Using (4.10) along with the propagator identities (A.1) listed in the Appendix at the end of the Paper, we find

$$
\begin{aligned}
\mathcal{A}_{f}^{\text {(grav) }}\left(p_{1}, p_{2} ; q\right)=-\frac{\pi}{2 k^{\prime} M}\left\{\left(3-\frac{q^{2}}{M^{2}}\right) \bar{u}\left(p_{1}-q\right) u\left(p_{1}\right) \bar{u}\left(p_{2}+q\right) u\left(p_{2}\right)\right. \\
+\frac{2\left(q^{2}+M^{2}\right)}{M\left(q^{2}-M^{2}\right)}\left(q_{\lambda} \bar{u}\left(p_{2}+q\right) \gamma^{\lambda} u\left(p_{2}\right) \bar{u}\left(p_{1}-q\right) u\left(p_{1}\right)\right. \\
\left.-q_{\lambda} \bar{u}\left(p_{1}-q\right) \gamma^{\lambda} u\left(p_{1}\right) \bar{u}\left(p_{2}+q\right) u\left(p_{2}\right)\right) \\
\left.+2 M \Omega_{\mu \nu}^{i j}(q) \epsilon_{\mu \lambda}^{i} \epsilon_{\nu \rho}^{j} \bar{u}\left(p_{1}-q\right) \gamma^{\lambda} u\left(p_{1}\right) \bar{u}\left(p_{2}+q\right) \gamma^{\rho} u\left(p_{2}\right)\right\}
\end{aligned}
$$

The amplitude (4.15) can be simplified using the Dirac equation (4.13) and the identity (A.7). We are interested in the small momentum limit of this amplitude, i.e. $q^{2} \rightarrow 0$. In that limit, the fermion bilinears appearing above become

$$
\bar{u}(p \pm q) u(p) \rightarrow 1 \quad, \quad \bar{u}(p \pm q) \gamma^{\mu} u(p) \rightarrow(2 p \pm q)^{\mu} / 2 m
$$

and we arrive at

$$
\mathcal{A}_{f}^{\text {(grav) }}\left(p_{1}, p_{2} ; q\right)=\frac{\pi}{2 k^{\prime} M}+\frac{3 \pi i}{k^{\prime} m^{2}} \frac{\epsilon_{\mu \nu \lambda} p_{1}^{\mu} p_{2}^{\nu} q^{\lambda}}{M^{2}}
$$

The imaginary, parity-odd part of the amplitude (4.17) does not have a singular pole term at $q^{2}=0$ like in the gauge theory case, so it does not really represent a genuine AharonovBohm interaction amplitude. The entire amplitude vanishes in the infrared limit $M \rightarrow \infty$ when the topologically massive gravity theory becomes topological Einstein gravity. This is similar to the effect of taking $k \rightarrow \infty$ in the Chern-Simons gauge amplitude (3.11). The "pole" structure here appears as $M^{2} \rightarrow 0$ (equivalently $\kappa \rightarrow 0$ ) when the gravitational Chern-Simons action in (2.2) dominates. In fact, the zero-mass limit of topologically massive gravity is equivalent to pure topological $S O(2,1)$ Chern-Simons gauge theory [8]. In this regime, where $M^{2} \rightarrow 0$ with $q^{2} \ll M^{2}$, the moduli space structure of the induced two-dimensional gravity theory disappears. The value $\Lambda=0$ of the boundary cosmological constant corresponds to a critical point of the three-dimensional quantum field theory, namely the point where the topologically massive gravity action exhibits local $(2+1)$-dimensional conformal invariance [1]. The induced spin can be identified from (4.17) as a quantity which is independent of the topological graviton mass scale, so that the singularity at the conformal symmetry point $M^{2}=$ 0 can be regarded as a kinematical pole corresponding to the usual non-dynamical singularity characteristic of a pure Chern-Simons gauge amplitude (with gauge group $S O(2,1)$ ). We can use these facts to interpret the imaginary part of (4.17) as an Aharonov-Bohm type amplitude, and comparing with (3.11) this identifies the gravitationally-dressed spin- $\frac{1}{2}$ weight

$$
\Delta_{1 / 2}^{(1)}=1 / 4
$$


which agrees with the coefficient of the $\mathcal{O}\left(1 / k^{\prime}\right)$ term in (3.7) for $j=1 / 2$. Later on we will describe another way of determining the anomalous conformal dimension from the parity-odd structure in (4.17).

The feature that the gravitational Chern-Simons term does not induce a singular momentum pole term owes to the fact that the quadratic form for the spin-connection in the topologically massive gravity action $(2.11)$ is non-degenerate. This is because the $\omega \wedge d e$ term in the Einstein action acts like a Proca mass term for a gauge field, thus removing all infrared singular pole terms. It does, however, induce a parity-odd structure indicative of the renormalization of the spin, where we naively interchange the roles $M^{2} \leftrightarrow q^{2}$ for the above correspondences. With this interchange the low-energy structure of topologically massive gravity begins to resemble that of topologically massive gauge theory [1], i.e. one with both a Yang-Mills kinetic term and a topological Chern-Simons term for the gauge fields. The structure of these amplitudes in topologically massive gravity is opposite to those of the gauge theory. This is another indication of the sort of duality that has been previously observed in [12] for the perturbative, infrared structure of these two topologically massive quantum field theories.

To explore this $q^{2}=0$ behaviour further, let us now consider the one-graviton exchange amplitude in the infrared limit

$$
\begin{aligned}
\mathcal{B}_{f}\left(p_{1}, p_{2} ; q\right)= & i \bar{u}\left(p_{1}-q\right) \mathcal{G}_{i}^{\mu}\left(p_{1}, p_{1}-q\right) u\left(p_{1}\right) D_{\mu \nu}^{i j}(q) \bar{u}\left(p_{2}+q\right) \mathcal{G}_{j}^{\nu}\left(p_{2}, p_{2}+q\right) u\left(p_{2}\right) \\
= & -i\left[4 \bar{u}\left(p_{1}-q\right) \gamma^{i} u\left(p_{1}\right) \bar{u}\left(p_{2}+q\right) \gamma^{j} u\left(p_{2}\right) p_{1}^{\mu} p_{2}^{\nu} D_{\mu \nu}^{i j}(q)+4 m^{2} D_{i j}^{i j}(q)\right. \\
& \left.\quad+2 m \bar{u}\left(p_{1}-q\right) \gamma^{i} u\left(p_{1}\right) p_{1}^{\mu} D_{\mu j}^{i j}(q)+2 m \bar{u}\left(p_{2}+q\right) \gamma^{j} u\left(p_{2}\right) p_{2}^{\nu} D_{i \nu}^{i j}(q)\right]
\end{aligned}
$$

where we have used the on-shell conditions $p^{2}=(p \pm q)^{2}=m^{2}$, the Dirac equation (4.13), and transversality of the free graviton propagator. In the small $q^{2}$ limit, using the propagator identities (A.14) listed in the Appendix we find

$$
\mathcal{B}_{f}\left(p_{1}, p_{2} ; q\right)=\frac{60 \pi m^{2}\left(q^{2}+M^{2}\right)}{k^{\prime} M q^{2}\left(q^{2}-M^{2}\right)}
$$

Thus the one-graviton exchange amplitude has only a parity-even part, and so it does not contribute to the anomalous spin of the fields. Furthermore, it has a singular pole structure at $q^{2}=0$, reflecting the fact that the original Einstein theory $(M \rightarrow \infty$ in (2.2) $)$ can be regarded as a topological Chern-Simons theory with gauge group $I S O(2,1)$. This topological Einstein theory contains no propagating degrees of freedom, which is why the characteristic pole at $q^{2}=0$ appears here, as in the case of the topological pure Chern-Simons gauge theory.

Finally, we consider the amplitude for the exchange of one ew boson,

$$
\mathcal{C}_{f}\left(p_{1}, p_{2} ; q\right)=i \bar{u}\left(p_{1}-q\right) \mathcal{W}_{i}^{\mu} u\left(p_{1}\right) E_{\mu \nu}^{i j}(q) \bar{u}\left(p_{2}+q\right) \mathcal{G}_{j}^{\nu}\left(p_{2}, p_{2}+q\right) u\left(p_{2}\right)
$$


Using the spin-connection propagator identities (A.1), (A.6) and (A.7) we find that the $q^{2} \rightarrow 0$ limit of the amplitude (4.21) becomes

$$
\mathcal{C}_{f}\left(p_{1}, p_{2} ; q\right)=\frac{3 \pi}{k^{\prime} M}\left(3+\frac{4 m}{M}\right)+\frac{4 \pi i}{k^{\prime} m^{2}} \frac{\epsilon_{\mu \nu \lambda} p_{1}^{\mu} p_{2}^{\nu} q^{\lambda}}{M^{2}}\left(\frac{q^{2}}{M^{2}}\right)
$$

The parity-odd term and the term proportional to the fermion mass $m$ in the parity-even part of the amplitude (4.22) come from the spin-connection part of the ew propagator $E_{\mu \nu}^{i j}(q)$ in (2.17), while the remaining parity-even piece comes from the $\beta e$ part of $E_{\mu \nu}^{i j}(q)$. Although the parity-odd piece in (4.22) has a similar structure as that which appears in the $\omega$-exchange amplitude, it is of order $q^{2} / M^{2}$ and thus vanishes in the infrared regime $q^{2} \ll M^{2}$ as compared to the amplitude (4.17), i.e. it vanishes in the correlated limit above in which we take first take $q^{2} \rightarrow 0$ and then identify the kinematical-type pole at $M^{2} \rightarrow 0$. This results from the manner in which the extra spin-connection term is convoluted in (2.15) with the spin matrix $\Sigma^{k l}$ to yield the transverse projection of $\Omega_{\mu \nu}^{i j}(q)$ in (2.17). Thus the we-exchange amplitude leads to $\mathcal{O}\left(q^{2} / M^{2}\right)$ corrections to the parity-odd, Aharonov-Bohm type amplitude determining the conformal weights and so, by definition, it yields no contribution to the induced spin at tree-level in perturbation theory. It would be interesting to further explore the (relativistic) structure of the parity-odd amplitudes above more carefully and hence study more precisely the interactions between topologically massive gravity and spinning matter fields, and also its potential relevence to the genuine gravitational Aharonov-Bohm effect [4, 5].

The above structures that are a result of the form of the gravitational propagators will play an important role in the higher-loop scattering amplitudes. The parity-odd structures from the graviton and $e \omega$ lines will always vanish at $q^{2} \rightarrow 0$, which is a result of the index contractions from the vertices formed by the kinetic terms of the matter fields coupled to the gravity fields [12]. These parity-odd pieces, which are a result of the covariant derivative relation between the $e$ and $\omega$ fields, are expected to vanish in order to recover the pure Einstein theory results. However, in diagrams with both graviton or ew and spin-connection lines, the parity-odd part of the spin-connection propagator will combine with the parity-even, singular part of the graviton or ew propagators, thus producing the required Aharonov-Bohm interaction terms. It is this sort of interplay between the $e$ and $\omega$ fields that will lead to a more complicated conformal dimension in higher loops than that anticipated from a naive gauge theory calculation. This sort of renormalization at higher-loop orders is exemplified in the gravitational dressing of an induced spin from the interaction of charged scalar fields with ordinary Chern-Simons gauge theory [12]. The parity-even parts of the graviton propagator $D_{\mu \nu}^{i j}(q)$ renormalize the parity-odd part of the Chern-Simons gluon propagator and conspire to yield the anticipated KPZ scaling at one-loop order (in the Einstein limit $M \rightarrow \infty$ ). The remaining combinations, however, vanish identically. The fact that such an interplay doesn't appear at tree-level here is the reason why there is no singular pole term in the $\omega$ - 
exchange amplitude, but it still does allow the identification of the correct KPZ induced spin as above. It would be interesting to extend this calculation to one-loop order and match the diagrammatic contributions with the $\mathcal{O}\left(1 / k^{\prime 2}\right)$ term in the KPZ formula. It would also be interesting to see how these higher-loop amplitudes behave in the two limits $M \rightarrow \infty$ and $M \rightarrow 0$ where topologically massive gravity is equivalent to topological Chern-Simons gauge theories (with gauge groups $I S O(2,1)$ and $S O(2,1)$, respectively).

\subsection{Vector Representations}

Finally, we couple to fields $\Phi^{(1)}$ in the defining, vector representation of $S O(2,1)$. The simplest Lorentz- and gauge-invariant model is the abelian topologically massive gauge theory

$S_{V}=\int_{\mathcal{M}}-\frac{1}{4 e^{2}}(\nabla A)^{*} \wedge \star \nabla A+S_{C S}^{[U(1)]}=\int_{\mathcal{M}} d^{3} x\left(-\frac{1}{4 e^{2}} \sqrt{g} g^{\mu \lambda} g^{\nu \rho} F_{\mu \nu} F_{\lambda \rho}+\frac{k}{8 \pi} \epsilon^{\mu \nu \lambda} A_{\mu} \partial_{\nu} A_{\lambda}\right)$

The Chern-Simons term is introduced to regulate the logarithmic infrared divergences in the pure Maxwell theory by giving the photons of the model a topological mass $m=k e^{2} / 4 \pi$. Since this term is topological, it does not couple to the spacetime metric and so all results below will be valid even in its absence. Now there is no coupling to the (torsion-free) connection $\omega$ because the spin-connection terms in the covariant derivatives in (3.8) cancel out by antisymmetry in the curvature $F_{\mu \nu}=\nabla_{\mu} A_{\nu}-\nabla_{\nu} A_{\mu}=\partial_{\mu} A_{\nu}-\partial_{\nu} A_{\mu}$. Thus the contribution to the conformal weight from the $\omega$ and we exchange amplitudes as described above is 0 .

It remains to check if there is any contribution from a parity-odd structure of the onegraviton exchange amplitude. Shifting the dreibein fields as above, the graviton-photonphoton vertex is 12

$$
\mathcal{F}_{i ; \nu \rho}^{\mu}(p ; q, r)=\frac{i}{2 e^{2}}\left[(r \cdot p) \delta_{i}^{\mu} \eta_{\nu \rho}-\delta_{i}^{\mu} r_{\nu} p_{\rho}+p^{\mu} r_{\nu} \eta_{i \rho}+2 r_{i} p_{\rho} \delta_{\nu}^{\mu}-2(r \cdot p) \delta_{\nu}^{\mu} \eta_{i \rho}-2 p^{\mu} r_{i} \eta_{\nu \rho}\right]
$$

where $p=q+r$, which leads to the exchange amplitude (Fig. 1 with the straight lines representing the photon fields and the wavy line depicting the dreibein field)

$$
\mathcal{B}_{v}\left(p_{1}, p_{2} ; q\right)=i e_{\nu}^{*}\left(p_{1}-q\right) \mathcal{F}_{i ; \mu \nu}^{\alpha}\left(p_{1}, p_{1}-q\right) e_{\mu}\left(p_{1}\right) D_{\alpha \beta}^{i j}(q) e_{\rho}^{*}\left(p_{2}+q\right) \mathcal{F}_{j ; \lambda \rho}^{\beta}\left(p_{2}, p_{2}+q\right) e_{\lambda}\left(p_{2}\right)
$$

where $e(p)$ are the on-shell polarization vectors for the photons in the transverse Landau gauge. These vectors describe the propagation of the single gauge degree of freedom in the quantum field theory $(4.23)$ and are determined from the equations of motion

$$
\left(\eta_{\mu \nu} \square+m \epsilon_{\mu \nu \lambda} \partial^{\lambda}\right) A^{\nu}=0
$$

in a covariant gauge $\partial_{\mu} A^{\mu}=0$. In momentum space, 4.26) leads to the on-shell equation

$$
m e_{\mu}(p)=-i \epsilon_{\mu \nu \lambda} p^{\nu} e^{\lambda}(p)
$$


which has solution

$$
e(p)=\frac{1}{\sqrt{2} m|\vec{p}|}\left(\begin{array}{c}
\vec{p}^{2} \\
p^{0} p^{1}-i m p^{2} \\
p^{0} p^{2}+i m p^{1}
\end{array}\right)
$$

where $(p)^{2}=\left(p^{0}\right)^{2}-\vec{p}^{2}=m^{2}$, and we have used the transverse normalizations

$$
p^{\mu} e_{\mu}(p)=0=e^{\mu}(p) e_{\mu}(p) \quad, \quad e^{\mu}(p) e_{\mu}^{*}(p)=-1
$$

In the limit $q^{2} \rightarrow 0$, using (4.28) and (4.29) we can simplify the amplitude (4.25) to

$$
\begin{array}{r}
\mathcal{B}_{v}\left(p_{1}, p_{2} ; q\right)=-\frac{i\left(q^{2}\right)^{2}}{16 e^{4}}\left[2 e_{i}^{*}\left(p_{1}-q\right) e^{\alpha}\left(p_{1}\right) D_{\alpha j}^{i j}(q)+2 e_{j}^{*}\left(p_{2}+q\right) e^{\beta}\left(p_{2}\right) D_{i \beta}^{i j}(q)\right. \\
\left.+4 e_{i}^{*}\left(p_{1}-q\right) e_{j}^{*}\left(p_{2}+q\right) e^{\alpha}\left(p_{1}\right) e^{\beta}\left(p_{2}\right) D_{\alpha \beta}^{i j}(q)+D_{i j}^{i j}(q)\right]
\end{array}
$$

Using (4.28) we have

$$
\epsilon_{\mu \lambda}^{j} e^{\mu}(p) e_{j}^{*}(p \pm q) q^{\lambda}=-\frac{i p \cdot q}{m}=\mp \frac{i q^{2}}{2 m}
$$

where $p=p_{1}$ or $p_{2}$, respectively. Using other similar such identities derived from (4.28) and (A.14), we find that all parity-odd contributions in (4.30) vanish in the infrared regime. The total amplitude in the limit $q^{2} \rightarrow 0$ is then

$$
\mathcal{B}_{v}\left(p_{1}, p_{2} ; q\right)=\frac{\pi}{2 k^{\prime} e^{4} M} q^{2}
$$

where the usual pole at $q^{2}=0$ has been cancelled in this case by the higher-momentum interactions from the vertex function (4.24). Thus the gravitational renormalization of the scaling dimension of the spin-1 fields here is

$$
\Delta_{1}^{(1)}=0
$$

which again agrees with the coefficient of the $\mathcal{O}\left(1 / k^{\prime}\right)$ term in the KPZ formula (3.7) for spin $j=1$.

We stress that the qualitative features of the above tree-level calculations are valid for any range of momentum $q$. The parity-odd parts of the graviton exchange amplitudes always vanish, because of the contractions of the free graviton propagator $D_{\mu \nu}^{i j}(q)$ with a conserved current, while the amplitudes involving the spin-connection lead to the required parity-odd structure identifying the conformal dimensions. These latter amplitudes do not contain the kinematic zero-momentum pole characteristic of Aharonov-Bohm scattering. However, the $q^{2} \rightarrow 0$ limits of all of these amplitudes are independent of the mass scale of the theory (modulo the $1 / M^{2}$ behaviour of the parity-odd parts), i.e. they hold independently of the size of the mass ratio $m / M$. It is this scale independence that is the remarkable feature of the $(2+1)$-dimensional gravitational dressing of the spins. This feature was also pointed out in [12]. In the next Section we shall present an alternative way of describing the spectrum of anomalous dimensions in topologically massive gravity. 


\section{$5 \quad$ KPZ Weights as $I S O(2,1)$ Anomalous Spin}

In the previous Section we demonstrated the role of the local $S O(2,1)$ spacetime symmetry group of topologically massive gravity in its relation to the quantum Liouville theory. It illustrates explicitly how the mysterious "hidden" $S L(2, \mathbb{R})$ Kac-Moody symmetry of twodimensional quantum gravity [14 arises as a dynamical property of the coupling of threedimensional gravity to sources. This realization of the gravitationally-dressed scaling weights is completely independent of the mass scale of the theory, but it requires tuning the topological graviton mass to a neighbourhood of the conformally-invariant point in the parameter space of the three-dimensional quantum field theory. This limit is required to properly identify the Aharonov-Bohm type amplitudes in the low-energy regime of the theory. The resulting scaling dimensions then appear as the usual induced fluxes responsible for the Aharonov-Bohm phase factors characteristic of the reduction of topologically massive gravity to topological $S O(2,1)$ Chern-Simons gauge theory.

The unusual limiting procedure required above suggests that we should look for an alternative way to identify the anomalous conformal dimensions which does not involve taking an unusual correlated low-energy limit as above. For this, we exploit the fact that we are really interpreting the scaling weights as the induced spin of the matter fields from their interaction with topologically massive gravity and consider the explicit renormalization of spin. The parity-odd structure of topologically massive gravity allows a very natural way of doing this in $(2+1)$-dimensions. As we shall see, this leads to a renormalized weight which is scale dependent and reduces to the anticipated KPZ scaling weights in the other topological limit of topologically massive gravity, namely the limit of pure Einstein gravity (equivalently $I S O(2,1)$ Chern-Simons gauge theory). In this way we will have a dynamical description of the scaling dimensions in the full parameter regime of topologically massive gravity which incorporates both of the fundamental symmetry groups $S O(2,1)$ and $I S O(2,1)$ of the three-dimensional quantum field theory, as well as the discrete parity-violating property of Chern-Simons quantum field theory. This illustrates the potential relevance of the geometric symmetries of the topological membrane approach to the gravity sector of string theory.

\subsection{Anomalous Magnetic Moment in Topologically Massive Grav- ity}

As discussed in [19], the geometry of a $(2+1)$-dimensional spacetime allows the identification of the induced fractional spin in an unambiguous way through the magnetic moment. The 
key feature is that the $(2+1)$-dimensional gamma-matrix identity (4.7) implies that the spin matrix generator on the space of spin- $\frac{1}{2}$ fields is given by

$$
\sigma^{\mu \nu} \equiv \frac{i}{2}\left[\gamma^{\mu}, \gamma^{\nu}\right]=-\epsilon^{\mu \nu \lambda} \gamma_{\lambda}
$$

and so the Gordon decomposition for the $(2+1)$-dimensional spinor current is

$$
\bar{u}(p-q) \gamma^{\mu} u(p)=\frac{(2 p-q)^{\mu}}{2 m} \bar{u}(p-q) u(p)-\frac{i}{2 m} \epsilon^{\mu \nu \lambda} q_{\lambda} \bar{u}(p-q) \gamma_{\nu} u(p)
$$

In the non-relativistic limit $q^{2} \rightarrow 0$ of the corresponding Dirac Hamiltonian, the first term in (5.2) represents the Coulomb charge interaction when the fermion fields are minimally coupled to an electromagnetic vector potential $A_{\mu}$ (see (4.16)). The other term represents the Pauli magnetic moment interaction in the usual way. In the non-relativistic limit, we see then that the quantity

$$
\varrho^{\mu}=-\frac{i}{2 m} \frac{\epsilon^{\mu \nu \lambda} p_{\nu} q_{\lambda}}{m}
$$

describes the usual magnetic moment structure in the Pauli interaction.

The function $\varrho^{\mu}$ is an axial vector, and is therefore odd under parity. The appearence of the magnetic moment structure in $(2+1)$-dimensions is therefore very natural for a parityviolating quantum field theory, such as Chern-Simons theory. It can be determined by examining the parity-odd structure of the one-particle irreducible vertex function $\Gamma_{\mu}(p, q)$ which is defined through the complete vertex function

$$
V_{\mu}(p, q) \equiv\left\langle\psi(p-q) A_{\mu}(q) \bar{\psi}(p)\right\rangle=S(p-q) i \Gamma_{\mu}(p, q) S(p)
$$

for the interaction of fermion fields minimally coupled to a background $U(1)$ gauge field $A_{\mu}$. Here $S(p)=\langle\psi(p) \bar{\psi}(-p)\rangle$ is the renormalized fermion propagator in momentum space. The identity (5.1) shows that the vertex function $\Gamma_{\mu}(p, q)$ can be decomposed into parity-even and parity-odd pieces which define the usual form factors by

$$
\Gamma_{\mu}(p, q)=\gamma_{\mu} \Gamma_{e}\left(q^{2}\right)+\frac{i}{2 m} \sigma_{\mu \nu} q^{\nu} \Gamma_{o}\left(q^{2}\right)
$$

The decomposition (5.5) holds in the gravitationally-dressed theory because of the discrete symmetries of the topologically massive gravity action. To actually compute the irreducible vertex function (5.5) we consider $\Gamma_{\mu}(p, q)$ to be contracted between Dirac spinors as $\bar{u}(p-$ $q) \Gamma_{\mu}(p, q) u(p)$. We shall not write the spinors explicitly but we freely use the Gordon relation (5.2). It implies that the renormalization of the magnetic moment structure (5.3) is determined by the low-energy limit of the sum $\Gamma_{e}\left(q^{2}\right)+\Gamma_{o}\left(q^{2}\right)$. However, the longitudinal form factor $\Gamma_{e}\left(q^{2}\right)$ can be absorbed into other renormalizations of the quantum field theory. This follows from the Ward-Takahashi identity

$$
q^{\mu} \Gamma_{\mu}(p, q)=\Sigma(p-q)-\Sigma(p)
$$


where $\Sigma(p)$ is the fermion self-energy operator. As proven in [12], the standard set of WardTakahashi identities holds individually for the radiative corrections due to gravity, because the gravity fields are themselves coupled to conserved matter currents in the gravitational interaction terms. The relation (5.6) shows that the longitudinal component of the vertex function (5.5) can be cancelled by the corresponding counterterms which renormalize the fermion mass, in addition to the $U(1)$ fermion charge.

Thus the only contribution from the vertex function to the magnetic moment structure $\varrho^{\mu}$ is effectively from the parity-odd form factor $\Gamma_{o}\left(q^{2}\right)$, and so we can identify the magnetic moment $\mu$ of the fermion fields as

$$
\mu \equiv \frac{g}{2 m} \Delta=-\frac{1}{2} \lim _{q^{2} \rightarrow 0} \Gamma_{o}\left(q^{2}\right)
$$

where we have explicitly incorporated the cancellation of the self-energy corrections to the external fermion lines with the parity-even component of the irreducible vertex function. Here we assume that the initial and final fermions are on-shell, $p^{2}=(p-q)^{2}=m^{2}$, and that they have gyromagnetic ratio $g=2$. The magnetic moment (5.7) can be used as an alternative definition of the renormalized (transmuted) spin $\Delta$. Note that in the infrared limit $q^{2} \rightarrow 0$, we can make the replacements (4.16) and compute the parity-odd form factor from

$$
\Gamma_{o}\left(q^{2}\right)=\frac{8 i m}{q^{2}\left(q^{2}-4 m^{2}\right)} \epsilon^{\mu \nu \lambda} p_{\nu} q_{\lambda} \Gamma_{\mu}(p, q)
$$

Thus we can use the above discussion to obtain an unambiguous definition of the renormalized spin $\Delta_{1 / 2}\left(k^{\prime}\right)$ for the interaction of fermion fields with topologically massive gravity. For this, we add to our previous gravitationally-dressed fermion action the term

$$
S_{A}=\int_{\mathcal{M}} d^{3} x \sqrt{g} g^{\mu \nu} \bar{\psi} \gamma_{\mu} A_{\nu} \psi
$$

where $A$ is a non-dynamical photon field. Then we compute the irreducible vertex function $\Gamma_{\mu}(p, q)$ for the renormalizations due to the gravitational dressing order by order in $1 / k^{\prime}$. Defining the form factors as above and using the relation (5.7), we can then identify the renormalized spin coefficients $\Delta_{1 / 2}^{(n)}$ and compare them with the iterative expansion of the KPZ formula. This gives a very natural definition of this induced spin for the spinor-coupled topologically massive gravity theory which exploits the explicit parity-odd structure characteristic of the usual identifications of the conformal dimensions. Furthermore, this alternative definition of the scaling weights avoids the unusual limiting procedures of the previous Section required to identify the spins using Aharonov-Bohm amplitudes. 


\subsection{One-loop Proper Vertex Function}

As an explicit example of the above approach, we shall now compute the total one-loop renormalization due to topologically massive gravity of the one-particle irreducible fermionfermion-photon vertex function. We use the usual expansions of the metric about the flat background to obtain the relevant vertices involving the background photon field at one-loop order. The fermion-fermion-photon vertex is $-i \gamma_{\mu}$, the $\bar{\psi} \psi A e$ vertex is

$$
\mathcal{H}_{\nu ; \mu}^{i}\left(p, p^{\prime} ; q ; r\right)=-i\left(\gamma_{\mu} \delta_{\nu}^{i}+\gamma^{i} \eta_{\mu \nu}+\gamma_{\nu} \delta_{\mu}^{i}\right)
$$

where $q+r=p-p^{\prime}$, and the $\bar{\psi} \psi$ Aee vertex function is

$$
\mathcal{H}_{\nu \lambda ; \mu}^{i j}\left(p, p^{\prime} ; q ; r, s\right)=-i\left(\gamma^{i} \delta_{\mu}^{j} \eta_{\nu \lambda}+\gamma^{j} \delta_{\nu}^{i} \eta_{\mu \lambda}+\gamma^{i} \delta_{\lambda}^{j} \eta_{\mu \nu}-\frac{1}{2} \gamma_{\mu} \eta_{\nu \lambda} \eta^{i j}+\frac{1}{2} \gamma_{\mu} \delta_{\nu}^{i} \delta_{\lambda}^{j}-\gamma_{\mu} \delta_{\lambda}^{i} \delta_{\nu}^{j}\right)
$$

with $q+r+s=p-p^{\prime}$.

The nine diagrams which contribute to the one-loop order renormalization of the irreducible vertex function $\Gamma_{\mu}(p, q)$ are shown in Fig. 2. The first diagram is the triangle graph from the exchange of the spin-connection field and it will be evaluated explicitly below. The next three Feynman graphs involving exchanges of the dreibein field do not contribute to the parity-odd part of the vertex function. This can be checked explicitly using the above Feynman rules and those in Section 4.2, and it follows from the general feature that this parity-odd structure is always cancelled from the coupling of the transverse dreibein fields to the conserved, dynamical gravitational matter currents [12]. The fifth diagram in Fig. 2 is the graviton tadpole graph and it is given explicitly by

$$
\Gamma_{\mu}^{[\bigcirc]}(p, q)=\int \frac{d^{3} k}{(2 \pi)^{3}} \mathcal{H}_{\nu \lambda ; \mu}^{i j} D_{i j}^{\nu \lambda}(k)
$$

After some algebra the parity-odd part of (5.12) is readily seen to vanish,

$$
\Gamma_{\mu}^{[\bigcirc]}(p, q)^{\text {odd }}=\frac{16 \pi i}{k^{\prime}} \epsilon_{\mu \nu \lambda} \gamma^{\lambda} \int \frac{d^{3} k}{(2 \pi)^{3}} \frac{k^{\nu}}{k^{2}\left(k^{2}-M^{2}\right)} \equiv 0
$$

after an appropriate dimensional regularization of the Feynman integral. The last four diagrams involving exchanges of the we fields can generally have finite contributions to $\Gamma_{o}\left(q^{2}\right)$ for non-zero $q^{2}$ due to the spin-connection propagator. However, because of the form of the coupling to the transverse graviton propagator and the transverse projection of $\Omega_{\mu \nu}^{i j}(q)$ (see Section 4.2), these contributions will vanish in the non-relativistic limit $q^{2} \rightarrow 0$. Again this is a feature of the coupling of the transverse gravitational fields to the conserved matter currents [12.

Thus, as anticipated on general grounds from the discussion of the previous two Sections, the low-energy limit of the parity-odd part of the vertex function (and hence the renormalization of the spin) at one-loop order is determined solely by the interaction of the fermion fields 

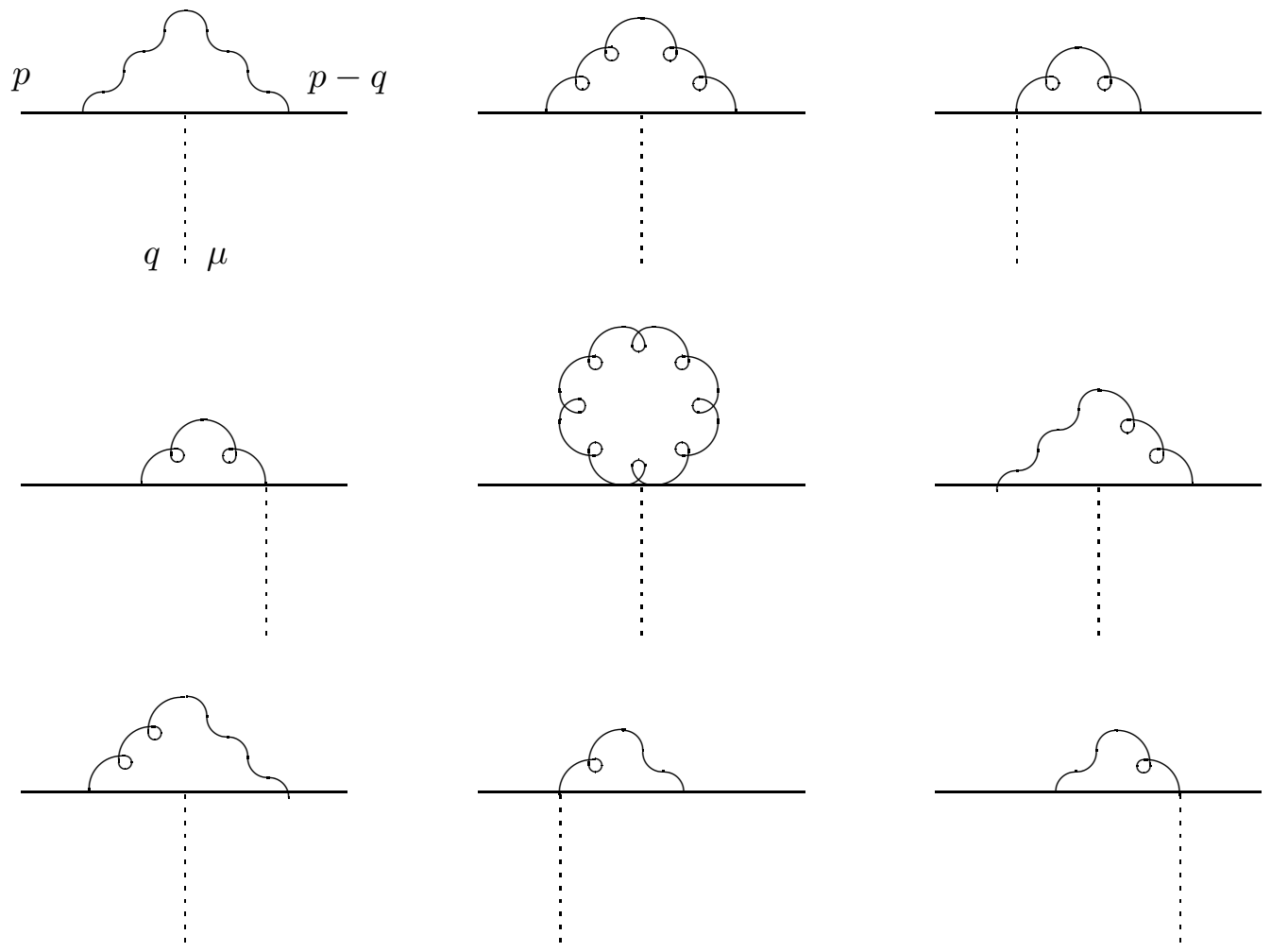

Figure 2: Feynman diagrams which contribute to the gravitational renormalization of the fermionfermion-photon vertex at one-loop order. Straight lines denote the fermion fields, wavy lines depict the spin-connection fields $\omega_{\mu}^{a}$, spiral lines represent the dreibein fields $e_{\mu}^{a}$, and dashed lines denote the background photon field $A_{\mu}$. 
with the spin-connection $\omega_{\mu}^{a}$. The triangle diagram for the exchange of one spin-connection field in Fig. 2 contributes the term

$$
\Gamma_{\mu}^{[\omega]}(p, q)=-\frac{4 \pi i}{k^{\prime}} \int \frac{d^{3} k}{(2 \pi)^{3}} \mathcal{W}_{j}^{\nu} S^{(0)}(p-q-k) \gamma_{\mu} S^{(0)}(p-k) \mathcal{W}_{i}^{\lambda} \Omega_{\lambda \nu}^{i j}(k)
$$

to the total one-loop irreducible vertex function, where

$$
S^{(0)}(p) \equiv\langle\langle\bar{\psi}(p) \psi(-p)\rangle\rangle=i\left(p_{\mu} \gamma^{\mu}-m\right)^{-1}=\frac{i}{p^{2}-m^{2}}\left(p_{\mu} \gamma^{\mu}+m\right)
$$

is the free fermion propagator in momentum space. We can simplify the terms in (5.14) by successively applying the $(2+1)$-dimensional gamma-matrix identity (4.7) and the on-shell conditions $p_{\mu} \gamma^{\mu}=(p-q)_{\mu} \gamma^{\mu}=-m, p^{2}=(p-q)^{2}=m^{2}$ and $p \cdot q=q^{2} / 2$ for the external particles (imagining, as mentioned above, that the vertex function (5.14) appears contracted between the two Dirac spinors $\bar{u}(p-q)$ and $u(p))$. This reduces (5.14) to the sum of a piece proportional to $\gamma_{\mu}$ and a piece proportional to $\sigma_{\mu \nu}$, as described in (5.5). We are interested in the parity-odd contributions, i.e. the terms in (5.14) proportional to $\epsilon_{\mu \nu \lambda}$. To extract these pieces, we simplify the vertex function using the spin-connection propagator identities listed in the Appendix. After a long and tedious algebraic calculation, we arrive at

$$
\begin{aligned}
& \Gamma_{o}^{[\omega]}\left(q^{2}\right)=\frac{8 \pi i m}{k^{\prime} M^{2} q^{2}\left(q^{2}-4 m^{2}\right)} \int \frac{d^{3} k}{(2 \pi)^{3}} \frac{1}{\left(k^{2}-2 k \cdot p\right)\left(k^{2}-2 k \cdot(p-q)\right)\left(k^{2}-M^{2}\right)} \\
&+\left\{\left(\epsilon_{\mu \nu \lambda} k^{\mu} p^{\nu} q^{\lambda}\right)^{2}\left[\left(\frac{3}{2 m}+\frac{1}{M}\right)\left(k^{2}-M^{2}\right)-M\right]\right. \\
&+\frac{q^{2}}{4}\left(4 m^{2}-q^{2}\right)\left[M\left(k^{2}-M^{2}\right)-\frac{1}{2 M}\left(3 M^{4}+\left(k^{2}\right)^{2}\right)+\frac{M^{2}}{4 m}[k \cdot(q-2 p-k)]\right. \\
&\left.+\frac{1}{4 m} k^{2}[k \cdot(10 p-3 q-3 k)]\right]+\left(m^{2}(k \cdot q)-\frac{q^{2}}{2}(k \cdot p)\right)\left[m\left(3 k^{2}+M^{2}\right)\right. \\
&+\frac{M}{2}[k \cdot(5 k-p-7 q)]-\frac{k^{2}}{2 M}[k \cdot(5 p+3 q)]-\frac{3}{2 M}\left(M^{4}-2\left(k^{2}\right)^{2}\right) \\
&\left.+\frac{k^{2}}{m}[k \cdot(5 p-2 q-3 k)]+\frac{M^{2}}{m}[k \cdot(q-2 p-k)]\right] \\
&+\frac{q^{2}}{2}[k \cdot(q-2 p)]\left[\frac{3 M}{4}\left(3 M^{2}-2 k^{2}\right)-m\left(k^{2}+6 M^{2}\right)+(k \cdot p)\left(\frac{k^{2}}{M}-\frac{7 M}{2}\right)\right. \\
&\left.\left.-\frac{9\left(k^{2}\right)^{2}}{4 M}+\frac{k^{2}}{2 m}[k \cdot(q-3 p+3 k)]+\frac{M^{2}}{2 m}[k \cdot(3 q-5 p+k)]\right]\right\}
\end{aligned}
$$

The $(2+1)$-dimensional momentum space integrations in (5.16) are absolutely convergent, and after some manipulation it is possible to cancel the higher powers of the loop momentum in the Feynman integral (5.16) with the denominator factors in its integrand. For example, the trivial identity

$$
2 k \cdot p=-\left(k^{2}-2 k \cdot p\right)+\left(k^{2}-M^{2}\right)+M^{2}
$$


reduces the tensorial rank of a given Feynman integration in (5.16) by 1 leaving a series of lower-rank integrals, most of which have fewer denominator factors in their integrands. Then all the loop momentum integrations can be carried out using the Feynman parametrizations

$$
\frac{1}{a b}=\int_{0}^{1} d x \frac{1}{[(1-x) b+x a]^{2}} \quad, \quad \frac{1}{a b c}=2 \int_{0}^{1} d x \int_{0}^{x} d y \frac{1}{[a y+b(x-y)+c(1-x)]^{3}}
$$

and the $(2+1)$-dimensional Feynman integral identities

$$
\begin{aligned}
& I^{(r)}\left(\alpha-p^{2}\right) \equiv \int \frac{d^{3} k}{(2 \pi)^{3}} \frac{1}{\left(k^{2}+2 k \cdot p+\alpha\right)^{r}}=\frac{\Gamma(r-3 / 2)}{8 \pi^{3 / 2} \Gamma(r)} \frac{1}{\left(\alpha-p^{2}\right)^{r-3 / 2}} \\
& \int \frac{d^{3} k}{(2 \pi)^{3}} \frac{k^{\mu}}{\left(k^{2}+2 k \cdot p+\alpha\right)^{s}}=-p^{\mu} I^{(s)}\left(\alpha-p^{2}\right) \\
& \int \frac{d^{3} k}{(2 \pi)^{3}} \frac{k^{\mu} k^{\nu}}{\left(k^{2}+2 k \cdot p+\alpha\right)^{s}}=\left(p^{\mu} p^{\nu}+\frac{\alpha-p^{2}}{2 s-5} \eta^{\mu \nu}\right) I^{(s)}\left(\alpha-p^{2}\right)
\end{aligned}
$$

which hold for $2 r>3$ and $2 s>5$. Using these identities and some further algebraic manipulations, after a long computation we arrive at the exact result for the spin-connection form factor

$$
\begin{aligned}
\Gamma_{o}^{[\omega]}\left(q^{2}\right)= & \frac{m}{336 k^{\prime} M^{2}\left(q^{2}-4 m^{2}\right)}\left\{32 M\left(4 m^{2}-q^{2}\right)\left[\frac{i F^{(1)}\left(q^{2}\right)}{\sqrt{-q^{2}}}-\frac{M^{2}}{2}\left(\frac{M}{m}-1\right) F^{(2)}\left(q^{2}\right)\right]\right. \\
& -8 M\left(12 m^{2} q^{2}-32 m^{4}+96 M m^{3}-24 M m q^{2}-\frac{M\left(q^{2}\right)^{2}}{m}+19 M^{3} m-\frac{6 M^{3} q^{2}}{m}\right. \\
& \left.-22 M^{2} m^{2}+7 M^{2} q^{2}\right) F^{(3)}\left(q^{2}\right)-\frac{8 M}{q^{2}}\left(4\left(q^{2}\right)^{2}-16 m^{2} q^{2}+64 M m^{3}-16 m M q^{2}\right. \\
& \left.-14 M^{2} q^{2}+46 m^{2} M^{2}-\frac{48 m^{2}\left(q^{2}\right)^{2}}{M^{2}}-32 M^{3} m+\frac{15 M^{3} q^{2}}{m}\right) F^{(4)}\left(q^{2}\right) \\
& +8|m|\left(\frac{2160 m^{2}}{M}-108 m+\frac{185 q^{2}}{m}-\frac{124 q^{2}}{M}\right)+\frac{2 M}{m}\left(4+\frac{23 M}{m}\right)\left(9 q^{2}-9 M m\right. \\
& \left.-4 m^{2}\right)-\frac{i}{\sqrt{-q^{2}}}\left(\frac{34\left(q^{2}\right)^{2}}{m}-92 m q^{2}-\frac{3252 m^{2} q^{2}}{M}-\frac{35\left(q^{2}\right)^{2}}{M}-\frac{288 m^{4}}{M}-400 m^{3}\right. \\
& \left.-8 m q^{2}-48 m^{2}+100 m M^{2}+18 M q^{2}-\frac{25 M^{2} q^{2}}{m}\right) \log \left(\frac{2|m|-i \sqrt{-q^{2}}}{2|m|+i \sqrt{-q^{2}}}\right) \\
& \left.+\frac{4 M}{m}\left(\frac{9 M^{2} q^{2}}{m^{2}}-\frac{9 M^{3}}{m}-4 M^{2}+32 m^{2}-8 q^{2}\right) \log \left(1+\frac{2 m}{M}\right)\right\}
\end{aligned}
$$


where $F^{(j)}\left(q^{2}\right)$ are the Feynman parametric integrals

$$
\begin{aligned}
F^{(1)}\left(q^{2}\right)=\int_{0}^{1} d x & \log \left(\frac{3 \sqrt{-q^{2}} x+2 i \sqrt{M^{2}(1-x)+\left(m^{2}+2 q^{2}\right) x^{2}}}{\sqrt{-q^{2}} x+2 i \sqrt{M^{2}(1-x)+m^{2} x^{2}}}\right) \\
F^{(2)}\left(q^{2}\right)=\int_{0}^{1} d x & \frac{x}{4 M^{2}(1-x)+\left(4 m^{2}-q^{2}\right) x^{2}} \\
& \times\left(\frac{3}{\sqrt{M^{2}(1-x)+\left(m^{2}+2 q^{2}\right) x^{2}}}-\frac{1}{\sqrt{M^{2}(1-x)+m^{2} x^{2}}}\right) \\
F^{(3)}\left(q^{2}\right)=\int_{0}^{1} d x & \frac{x^{2}}{4 M^{2}(1-x)+\left(4 m^{2}-q^{2}\right) x^{2}} \\
& \times\left(\frac{3}{\sqrt{M^{2}(1-x)+\left(m^{2}+2 q^{2}\right) x^{2}}}-\frac{1}{\sqrt{M^{2}(1-x)+m^{2} x^{2}}}\right) \\
F^{(4)}\left(q^{2}\right)=\int_{0}^{1} d x & \frac{1}{4 M^{2}(1-x)+\left(4 m^{2}-q^{2}\right) x^{2}} \\
& \times\left(\frac{M^{2}(1-x)+\left(q^{2}+2 m^{2}\right) x^{2}}{\sqrt{M^{2}(1-x)+m^{2} x^{2}}}-2 \sqrt{M^{2}(1-x)+\left(m^{2}+2 q^{2}\right) x^{2}}\right)
\end{aligned}
$$

The integrated forms of $F^{(j)}\left(q^{2}\right)$ are quite complicated and not very informative for generic values of $q^{2}$. However, in the momentum regimes of interest to be discussed below, they can be evaluated in terms of simple algebraic forms.

The vertex function (5.20) is finite in the infrared limit and at $q^{2} \rightarrow 0$ we find

$$
\begin{aligned}
\Gamma_{o}^{[\omega]}(0 ; M)= & -\frac{1}{672 k^{\prime} M^{2} m}\left\{M \log \left(1+\frac{2 m}{M}\right)\left[\frac{65 M^{2}}{m}-448 M-192 m-\frac{62 M^{3}}{m^{2}}\right]\right. \\
& +\frac{M}{M+2 m}\left[256 m^{2}-896 M m-\frac{88 M^{3}}{m}+84 M^{2}\right] \\
& \left.+70 M m+\frac{8784 m^{3}}{M}-232 m^{2}-\frac{212 M^{3}}{m}-178 M^{2}\right\}
\end{aligned}
$$

where for definiteness we have taken $m>0$. Note that the finiteness of the irreducible vertex function at $q^{2}=0$ confirms the general expectations of the infrared finiteness of topologically massive gravity in the Landau gauge [2, 3]. There are two different mass regimes of interest. The first is the regime of a heavy graviton field with mass $M>m$ and the other is that of a heavy fermion field of mass $m>M$. The topological Einstein limit of the gravity theory is the extreme first regime when $M \rightarrow \infty$, and taking this limit in (5.22) yields

$$
\lim _{M \rightarrow \infty} \Gamma_{o}^{[\omega]}(0 ; M)=-\frac{1}{2 k^{\prime} m}
$$

Comparing with (5.7) and incorporating the tree-level (bare) vertex function (see (5.2) ) we 
find that the total magnetic moment including the one-loop anomalous contribution is

$$
\mu^{(1)}=\frac{2}{2 m}\left(\frac{1}{2}+\frac{1}{4 k^{\prime}}\right)
$$

which identifies the total one-loop order gravitationally dressed $\operatorname{spin} \Delta_{1 / 2}^{(1)}\left(k^{\prime}\right)=1 / 2+1 / 4 k^{\prime}$, as predicted by the KPZ formula. Thus in the limit $M \rightarrow \infty$ where topologically massive gravity becomes topological Einstein gravity, the magnetic moment structure generated via the gravitational renormalizations by the spin-connection field yield another definition of the induced spin of the charged spinor fields which coincides with the predictions of quantum Liouville theory.

In the extreme second regime where $M \ll m$ we find

$$
\lim _{M \rightarrow 0} \Gamma_{o}^{[\omega]}(0 ; M)=-\frac{183}{14 k^{\prime} m}\left(\frac{m}{M}\right)^{3}
$$

Just like the tree-level interaction amplitudes, the one-loop vertex function diverges in the limit $M \rightarrow 0$ when the topological Einstein term in the gravity action becomes irrelevant and the gravity theory is equivalent to an $S O(2,1)$ Chern-Simons gauge theory (and possesses local conformal symmetry). In general, the $M$-dependent vertex function (5.22) determines an anomalous dimension $\Delta(M / m)$ which depends on the mass scale of the fermion-coupled topologically massive gravity theory. Since the natural bulk scale $M^{2} \propto \kappa^{2}$ of three-dimensional gravity coincides with the natural scale $\Lambda$ of the induced Liouville theory, this can be taken as a definition of scale dependent KPZ conformal dimensions, which are otherwise not predicted by the two-dimensional KPZ theory.

After some calculation we also find that $\Gamma_{o}^{[\omega]}\left(q^{2}\right) \rightarrow 0$ at $q^{2} \rightarrow \infty$. This means that the bare magnetic moment (and hence the bare spin $\frac{1}{2}$ ) of the fermion fields is not renormalized by the gravitational interaction at short distance scales. On the other hand, the long-range effects of the gravitational field do lead to an anomalous magnetic moment. However, it is only in the topological limit $M \rightarrow \infty$ that we obtain the expected Liouville dressed spin and the correct gyromagnetic ratio $g=2$ for the fermion fields. For finite values of $M$, the vertex function (5.22) can also be thought of as defining a scale dependent gyromagnetic ratio $g(\Lambda)=g(M / m)$ for the fermion fields which reduces to the canonical value $g=2$ at large-distance (i.e. topological) scales. The observables of matter-coupled topologically massive gravity in this infrared limit are associated with those of string theory in the critical dimension when the induced gravitational anomaly vanishes. However, since the anomalous spin in the fermion-coupled topologically massive gravity theory was shown in the previous Section to also coincide with the KPZ formula in the ultraviolet limit $M \rightarrow 0$, the qualitative features of our results are in effect independent of the topological graviton mass scale. They do not depend on which regime of the theory we are in and therefore hold for the full induced 
Liouville gravity theory as well. This is the important and somewhat surprising feature of the correspondence here between the two- and three-dimensional theories. It is interesting that these two regimes of topologically massive gravity actually represent two different symmetry points in the moduli space of three-dimensional quantum gravity theories. Either symmetry group leads to the appropriate renormalization of spin predicted by the KPZ theory. As we discuss in the next Section, the more natural appearence of the KPZ weights at Poincaréinvariant point corresponds to the fact that at this point in the moduli space the threedimensional quantum gravity theory coincides in some sense with topological gravity.

It would be interesting to extend the above cumbersome calculation (as well as those of the previous Section) to higher-loop orders. This would serve as a non-trivial check of the relationship between quantum Liouville theory and topologically massive gravity. It would also illustrate how the different gravity fields of the three-dimensional theory conspire to correctly renormalize spin beyond that which can be predicted based on a naive tree-level calculation. Such calculations would also provide further information about the seemingly mysterious scale dependences of the basic observables, for instance if there is any renormalization giving $g-2 \neq 0$ at higher-loop orders and hence leading to other gravitational renormalizations of the (anomalous) fermion magnetic moment. It would be interesting to use this scale dependence to further illustrate the precise roles of the symmetry groups of the three-dimensional gravity theory in relation to the induced two-dimensional quantum gravity theory.

\section{Induced Spin and Phases of Three-dimensional Quan- tum Gravity}

In this final Section we shall discuss some puzzles concerning induced spin in topologically massive gravity, and present some conjectures about how these unusual features of the threedimensional theory could be related to different phases of the Liouville theory (1.1). Our first observation concerns the other branch of the KPZ scaling relations (1.4), which is related to the solution (1.5) that vanishes at $\Delta_{0}=0$ by

$$
\Delta_{+}=-\left(k^{\prime}+1\right)-\Delta_{-}
$$

This solution of the KPZ formula diverges in the asymptotic limit $k^{\prime} \rightarrow \infty$. As is well-known, the KPZ theory is only valid for two-dimensional quantum gravity coupled to matter fields of central charge $c \leq 1$, in which case the canonical choice of dressed scaling dimension $\Delta_{-}$is taken. The other choice (6.1) for the dressed weights corresponds to changing the gravitational dressing in the tachyon operator term in (1.1) from $\mathrm{e}^{\alpha_{+} \phi}$ to $\mathrm{e}^{\alpha_{-} \phi}$, where

$$
4 \alpha Q=-2-\alpha^{2}
$$


It has been argued in [20] that this change of branch in the KPZ scaling relations effectively couples two-dimensional quantum gravity to matter fields of central charge $c>1$, which are to be thought of as embedding the world-sheet into a "crumpled" phase, rather than a smooth phase. In this phase the surfaces which dominate the string partition function are fragmented into trees of "baby universes", in each of which the usual $c<1$ behaviour is exhibited. This second phase of two-dimensional quantum gravity is known as the branched polymer phase, wherein the typical objects that contribute to the string partition function at large distance scales are tree-like objects, rather than the conventional two-dimensional surfaces which dominate in the stringy phase.

From a dynamical point view it is interesting that the perturbative regime of topologically massive gravity automatically selects the branch $\Delta_{-}$of the KPZ equations. In fact, as discussed in [5], the perturbative approach that we have presented can only yield this branch, because the effective coupling constant of the three-dimensional theory is $1 / k^{\prime}$. This same sort of argument is used in quantum Liouville theory to select this branch, because it is only for that choice that the semi-classical limit exists in the weak-field approximation $(c \gg 1)$. In particular, it is impossible to reach the conformally-invariant point in the parameter space of three-dimensional quantum gravity $\left(k^{\prime} \rightarrow \infty\right)$ with the branch (6.1). However, there is another sort of induced spin in topologically massive gravity which could be related to this other phase of the two-dimensional gravity theory. This induced spin is due to a purely topological feature of the effective three-dimensional spacetime and it is directly related to the gravitational analog of the Aharonov-Bohm effect $[$ due to the mass-energy generated by sources. This effect is most natural in three-dimensional gravity, where the vacuum Einstein equations $R_{\mu \nu}=0$ imply that locally the curvature vanishes, i.e. $R_{\mu \nu \lambda \rho}=0$. Thus outside of the support of matter fields the spacetime is flat, but, as shown in [21, globally the theory can exhibit non-trivial effects. In contrast to the four-dimensional case, the field equations imply exactly the classical equations of motion of particle singularities. As discussed in [22], a source of mass $m$ and spin $\Delta_{0}$ gives a helical time structure for the effective spacetime which can lead to interesting quantum mechanical effects. Furthermore, the spacetime has a locally flat conical geometry which shifts the angular momentum spectrum leading to an induced spin. Such a spacetime is characterized by a time interval $t \in\left[0,4 \kappa^{-1} \Delta_{0}\right]$ and a plane polar angle range $\phi \in\left[0,2 \pi-4 \kappa^{-1} m\right]$. Thus a point spinning particle induces a non-static spacetime with a curvature singularity at the origin (corresponding to a non-trivial holonomy about the origin) and also a torsion singularity. It is possible to measure the mass $m$ (per unit length) through the gravitational Aharonov-Bohm effect. This effect is of order $\kappa^{-1} m$.

In topologically massive gravity, the Einstein field equations are replaced by the EinsteinCotton equations

$$
\sqrt{g} R_{\mu \nu}+M^{-1} C_{\mu \nu}=-\kappa^{-1} \sqrt{g} T_{\mu \nu}
$$


where $T_{\mu \nu}$ is the energy-momentum tensor of a matter source and

$$
C_{\mu \nu}=\epsilon_{\mu \lambda \rho} \nabla^{\lambda}\left(R_{\nu}^{\rho}-\frac{1}{4} \delta_{\nu}^{\rho} R\right)
$$

is the trace-less, symmetric Cotton tensor density which is conserved $\left(\nabla^{\mu} C_{\mu \nu}=0\right)$ and conformally-invariant $\left(C_{\mu \nu}\right.$ is the three-dimensional analog of the Weyl tensor). The static and circularly symmetric solutions of the topologically massive gravity field equations (6.3) for a point particle of mass $m$ and spin $\Delta_{0}$ are [4]

$$
d s^{2} \equiv h_{\mu \nu}(x) d x^{\mu} \otimes d x^{\nu}=N(r) d t \otimes d t+\varphi(r) d \vec{x} \otimes d \vec{x}+d t \otimes d \vec{x} \times \overrightarrow{\partial W}(r)
$$

where $h_{\mu \nu} \equiv g_{\mu \nu}-\eta_{\mu \nu}$ and

$$
\begin{aligned}
& W(r)=-\frac{M^{-1}}{2 \pi \kappa}\left(m+M \Delta_{0}\right)\left(\log (M r)+K_{0}(M r)\right) \\
& \varphi(r)=\frac{1}{2 \pi \kappa}\left(m+M \Delta_{0}\right) K_{0}(M r)+\frac{m}{\pi \kappa} \log (M r) \\
& N(r)=\frac{1}{2 \pi \kappa}\left(m+M \Delta_{0}\right) K_{0}(M r)
\end{aligned}
$$

with $K_{0}(x)$ the irregular modified Bessel function of order 0 which has the asymptotic behaviours $K_{0}(x) \sim-\log x$ for $x \rightarrow 0$ and $K_{0}(x) \sim x^{-1 / 2} \mathrm{e}^{-x}$ for $x \rightarrow \infty$. These solutions are only valid for a weak-field metric (i.e. in the linearized approximation to the full non-linear theory).

The spatial part of the metric at infinity (corresponding to the infrared limit of topologically massive gravity) is

$$
\lim _{r \rightarrow \infty} \varphi(r)=\frac{m}{\pi \kappa} \log (M r)
$$

so that the solution (6.6) represents an asymptotically conical space whose angular deficit range exceeds $2 \pi$. An exact solution in the full non-linear theory with this asymptotically conical structure is possible only when $m+M \Delta_{0}=0$, in which case the above solution has the same form as that generated by a spinless point mass in pure Einstein gravity. In the linearized approximation, it is possible to construct the Noether generators using the above field equations, and one finds in the adiabatic limit that the canonical angular momentum of the particle is shifted by an amount

$$
\Delta_{\text {ind }}=-\frac{m^{2}}{32 \pi^{2} \kappa^{2}} k^{\prime}=-\frac{2}{k^{\prime}}\left(\frac{m}{M}\right)^{2}
$$

which corresponds to an induced spin of the particle generated by its interaction with the topologically massive gravity field. The quantity $m / M$ measures the amount of gravitational flux generated by the point mass $m$. Thus the induced spin in this non-perturbative, weak-field approach is scale dependent and has the same qualitative properties as the spin determined 
by the vertex function (5.25) in the ultraviolet regime of the topologically massive gravity theory. It is only on mass scales of the order of the topological graviton mass that we obtain an induced spin resembling the Liouville dressed conformal dimension arising from the perturbative regime of the three-dimensional theory.

The induced spin (6.8), which is non-vanishing even for spinless particle sources $\left(\Delta_{0}=0\right)$ will be more complicated in the full non-linear theory, but it is always determined as some non-perturbative dynamical effect in the theory. This induced spin comes from a gravitational analog of the Aharonov-Bohm effect since it is determined by the gravitational flux generated by the mass $m$. The linearized approximation in this approach is the analog of the tree-level perturbative calculations that we presented earlier. The fact that the induced spin (6.8) is non-zero for spinless particles suggests that it could be related to the non-perturbative branch $\Delta_{+}$of the KPZ scaling relations discussed above. It would be interesting to explore this potential relationship further, and also to examine how to relate to topologically-induced spins (6.8), which are induced by identical particle exchange in the asymptotic, field-free region of the spacetime, with the perturbative spins that we derived before, which arise from identical particle exchange where the particles interact gravitationally with each other in a flat space-time (without conical singularities). The charged particles act as sources for the gravitons which renormalize the bare spin of the particles. It is unclear how to relate the lowenergy quantum field theoretical amplitudes obtained in this Paper to the classical conical structure of spacetime [5]. Various solutions to the field equations exhibiting such singularity structures have been obtained recently in [23], and it would be interesting to connect these structures with the polymer structures dominating $c>1$ string theory.

The topological nature of the induced spin (6.8) also suggests that it could be related to some more direct topological property of three-dimensional gravity. One candidate is threedimensional topological gravity, where the dreibein and spin-connection fields are taken as independent variables $\left(S_{\lambda} \equiv 0\right.$ in (2.2) $)$. The classical equations of motion of this theory are $R=\nabla e^{a}=0$, so that at tree-level the induced spins in topological gravity will coincide with those above in topologically massive gravity. This theory can be written as a topological ISO $(2,1)$ Chern-Simons gauge theory and it was shown in [24] that a similar solution to that given above is obtained exactly in this full non-linear theory (and not just when $m+M \Delta_{0}=0$ ). This theory has no dynamical degrees of freedom, and thus it may be related to the $c>1$ phase of the quantum Liouville theory. Notice that in three-dimensional topological gravity one expects to obtain scattering amplitudes with kinematical poles characteristic of the AharonovBohm effect, which is why the KPZ weights were more naturally identified before using the Poincaré symmetry of the three-dimensional quantum field theory.

The other possibility is that the $\Delta_{+}$branch of the KPZ formula is reached in the topological phase of topologically massive gravity [8], i.e. the phase wherein $\left\langle e_{\mu}^{a}\right\rangle=0$ and there is no 
background spacetime. In this phase one cannot diagonalize the quadratic form of the bulk part of the action to find the propagators 8,12 , because there are only two gauge groups in the model (diffeomorphisms and local $S O(2,1)$ rotations) while there are three fields $(\beta, \omega$ and $e$ ) that require gauge-fixing. Thus the quadratic approximation does not exist and perturbation theory breaks down. Furthermore, when $\left\langle e_{\mu}^{a}\right\rangle=0$ the local $S O(2,1)$ symmetry is unbroken and both space-time and tangent space rotations individually preserve the symmetry of the ground state. It could be that this phase of the gravity theory (2.2) is intimately related to $c>1$ string theory. Note that there are actually several such topological phases, depending on how degenerate the dreibein field is. The intermediate phases occur when $\left\langle e_{\mu}^{a}\right\rangle \neq 0$, but $\operatorname{det}_{\mu, a}\left[\left\langle e_{\mu}^{a}\right\rangle\right]=0$. The number of different ways that this degeneration can occur depends on the topology of the underlying 3-manifold $\mathcal{M}$. It would be interesting to use the topological membrane approach in this way to model the fractal baby universe structure of the branched polymer phase of two-dimensional quantum gravity.

Some evidence for such topological interpretations lies in the fact that for the $\Delta_{-}$gravitational dressing, the puncture operator on the worldsheet $\Sigma$ is the local tachyon vertex operator $\mathrm{e}^{\alpha_{+} \phi}$ which creates microscopic loops on the worldsheet. This corresponds to the perturbative phase of the three-dimensional theory in which there are local, propagating graviton degrees of freedom which are exchanged by charged particles moving in the bulk $\mathcal{M}$. When the gravitational dressing is changed to the $\Delta_{+}$branch, it is the non-local, smeared tachyon vertex operator $\int_{\Sigma} d^{2} z \mathrm{e}^{\alpha_{-} \phi}$ which creates macroscopic loops on $\Sigma$. This is a global area form which corresponds to a topological phase of the model in which there are no propagating degrees of freedom at all. The distinction in two-dimensions between microscopic states corresponding to local operators and macroscopic states follows from the fact that the metric is a dynamical variable of the quantum Liouville theory. The dressing of the tachyon operator by a macroscopic state is not merely a local disturbance to the surface, because it creates a macroscopic hole and tears the surface apart. Integrating over all such contributions in the string partition function leaves all but a microscopic (of the order of the cutoff) fraction of the worldsheet with holes. Thus the surface deteriorates and the resulting model is in the branched polymer phase discussed above. From the three-dimensional point of view, the integrated tachyon vertex operators correspond to smeared-out particle worldlines (see Section 1) wherein the graviton exchanges cannot be described perturbatively. Thus the three-dimensional description of quantum Liouville theory has the potential of even providing a geometrical approach to the mysterious $c>1$ phase of string theory. It would be interesting to develop some alternative non-perturbative approach, such as a connection with the conical structure of the three-dimensional spacetime (and hence the gravitational Aharonov-Bohm effect), to describe this phase. 


\section{Acknowledgements}

We thank J. Wheater for helpful discussions. The work of R.J.S. was supported in part by the Natural Sciences and Engineering Research Council of Canada.

\section{Appendix Gravitational Propagator Identities}

In this Appendix we summarize some identities for the various gravitational propagators that are used throughout this Paper. The relevant formulas for the spin-connection propagator are as follows:

$$
\begin{aligned}
& \Omega_{i \nu}^{i j}(q)=-\frac{1}{2 M} \Lambda_{\nu}^{j}(q)+i \epsilon_{\nu \lambda}^{j} q^{\lambda} \frac{q^{2}+M^{2}}{2 M^{2}\left(q^{2}-M^{2}\right)} \\
& \Omega_{\mu j}^{i j}(q)=-\frac{1}{2 M} \Lambda_{\mu}^{i}(q)-i \epsilon_{\mu \lambda}^{i} q^{\lambda} \frac{q^{2}+M^{2}}{2 M^{2}\left(q^{2}-M^{2}\right)} \\
& \Omega_{i j}^{i j}(q)=\frac{1}{2 M}\left(\frac{q^{2}}{M^{2}}-3\right) \\
& \Omega_{\lambda i}^{i j}(q)=\frac{1}{q^{2}-M^{2}}\left(\frac{q^{2}-3 M^{2}}{2 M} \Lambda_{\lambda}^{j}(q)+i \epsilon_{\lambda \rho}^{j} q^{\rho}\right)+\frac{1}{2 M^{3}}\left(2 M^{2} \delta_{\lambda}^{j}-\frac{2\left(q^{2}-3 M^{2}\right)}{M^{2}} q_{\lambda} q^{j}\right) \\
& \Omega_{j \nu}^{i j}(q)=\frac{1}{q^{2}-M^{2}}\left(\frac{q^{2}-3 M^{2}}{2 M} \Lambda_{\nu}^{i}(q)-i \epsilon_{\nu \rho}^{i} q^{\rho}\right)+\frac{1}{2 M^{3}}\left(2 M^{2} \delta_{\nu}^{i}-\frac{2\left(q^{2}-3 M^{2}\right)}{M^{2}} q^{i} q_{\nu}\right) \\
& \Omega_{j i}^{i j}(q)=-\frac{1}{M^{3}\left(q^{2}-M^{2}\right)}\left(6 M^{2}\left(q^{2}-2 M^{2}\right)+\frac{\left(q^{2}\right)^{2}}{M^{2}}\left(q^{2}-3 M^{2}\right)\right) \\
& {\left[\eta_{i j} \Omega_{\lambda \nu}^{i j}(q)\right]^{\text {odd }}=\frac{i\left(q^{2}-2 M^{2}\right)}{M^{2}\left(q^{2}-M^{2}\right)} \epsilon_{\lambda \nu \rho} q^{\rho} \quad, \quad\left[\eta^{\mu \nu} \Omega_{\mu \nu}^{i j}(q)\right]^{\text {odd }}=\frac{i}{q^{2}-M^{2}} \epsilon^{i j \lambda} q_{\lambda}} \\
& q^{\lambda} \Omega_{\mu \lambda}^{i j}(q)=-\frac{1}{2 M^{3}}\left(q_{\mu} q^{i} q^{j}+M^{2} \delta_{\mu}^{i} q^{j}+2\left(q^{2}-M^{2}\right) \eta^{i j} q_{\mu}+2 q^{2} \delta_{\mu}^{j} q^{i}+i M \epsilon_{\mu}^{i \lambda} q_{\lambda} q^{j}\right) \\
& \epsilon_{i \rho}^{\mu} \Omega_{\mu \nu}^{i j}(q)=\frac{q^{\mu}}{M\left(q^{2}-M^{2}\right)}\left(\epsilon_{\mu \rho}^{j} q_{\nu}-\epsilon_{\mu \nu \rho} q^{j}\right)-\frac{i}{M^{2}} \eta_{\rho \nu} q^{j} \\
& \epsilon_{j \rho}^{\nu} \Omega_{\mu \nu}^{i j}(q)=\frac{i}{M^{2}} q^{i} \eta_{\mu \rho}-\frac{1}{M} \epsilon_{\mu \rho}^{i}+\frac{1}{2 M^{3}}\left(\epsilon_{\rho}^{\nu i} q_{\mu} q_{\nu}-3 \epsilon_{\mu \rho}^{\nu} q^{i} q_{\nu}\right)
\end{aligned}
$$




$$
\begin{aligned}
& {\left[\epsilon_{\mu i}{ }^{\nu} \Omega_{\lambda \nu}^{i j}(q)\right]^{\text {odd }}=\frac{1}{2 M\left(q^{2}-M^{2}\right)}\left(\left(3 M^{2}-q^{2}\right) \epsilon_{\mu \lambda}{ }^{j}+\frac{2\left(q^{2}-2 M^{2}\right)}{M^{2}}\left[\epsilon_{\mu i}{ }^{j} q^{i} q_{\lambda}+\epsilon_{\mu \lambda}{ }^{\nu} q_{\nu} q^{j}\right]\right)} \\
& {\left[\epsilon_{\mu i j} \Omega_{\lambda \nu}^{i j}(q)\right]^{\text {odd }}=\frac{3 M^{2}-q^{2}}{2 M\left(q^{2}-M^{2}\right)} \epsilon_{\mu \lambda \nu}-\frac{1}{M\left(q^{2}-M^{2}\right)}\left(\epsilon_{\mu i \nu} q^{i} q_{\lambda}+\epsilon_{\mu \lambda j} q^{j} q_{\mu}\right)} \\
& {\left[\epsilon_{\mu}^{\lambda \nu} \Omega_{\lambda \nu}^{i j}(q)\right]^{\text {odd }}=\frac{3 M^{2}-q^{2}}{2 M\left(q^{2}-M^{2}\right)} \epsilon_{\mu}^{i j}-\frac{1}{M\left(q^{2}-M^{2}\right)}\left(\epsilon_{\mu}^{\lambda j} q_{\lambda} q^{i}+\epsilon_{\mu}^{i \nu} q_{\nu} q^{j}\right)} \\
& {\left[\epsilon_{\mu}^{\lambda}{ }_{j}^{\lambda} \Omega_{\lambda \nu}^{i j}(q)\right]^{\text {odd }}=\frac{q^{2}+M^{2}}{2 M\left(q^{2}-M^{2}\right)} \epsilon_{\mu \nu}^{i}-\frac{1}{M\left(q^{2}-M^{2}\right)}\left(\epsilon_{\mu \nu}^{\lambda} q_{\lambda} q^{i}+\epsilon_{\mu j}^{i} q_{\nu} q^{j}\right)} \\
& {\left[\epsilon^{\lambda}{ }_{i \beta} \epsilon^{\nu}{ }_{j \mu} \Omega_{\lambda \nu}^{i j}(q)\right]^{\text {odd }}=-\frac{i}{M^{2}} \epsilon_{\beta j \mu} q^{j}}
\end{aligned}
$$

where [ • ] dedd denotes the parity-odd part of the given tensor function, i.e. the piece proportional to $\epsilon_{\mu \nu \lambda}$. We have also made use of the following identities for the graviton propagator:

$$
D_{i \nu}^{i j}(q)=D_{\nu i}^{j i}(q)=\frac{i}{\kappa}\left(\frac{q^{2}+M^{2}}{2 q^{2}\left(q^{2}-M^{2}\right)} \delta_{\nu}^{\perp j}(q)+i M \frac{\epsilon_{\nu \lambda}{ }^{j} q^{\lambda}}{q^{2}\left(q^{2}-M^{2}\right)}\right) \quad, \quad D_{i j}^{i j}(q)=\frac{i}{\kappa} \frac{q^{2}+M^{2}}{q^{2}\left(q^{2}-M^{2}\right)}
$$




\section{References}

[1] S. Deser, R. Jackiw and S. Templeton, Ann. Phys. 140 (1982), 372

[2] S. Deser and Z. Yang, Class. Quant. Grav. 7 (1990), 1603

[3] B. Keszthelyi and G. Kleppe, Phys. Lett. B281 (1992), 33

[4] S. Deser, Phys. Rev. Lett. 64 (1990), 611; Class. Quant. Grav. 9 (1992), 61;

S. Deser and J.G. McCarthy, Nucl. Phys. B344 (1990), 747;

Y.M. Cho and D.H. Park, Phys. Rev. D43 (1991), 1421; D49 (1994), 6269;

M. Reuter, Phys. Rev. D44 (1991), 1132;

M.E. Ortiz, Nucl. Phys. B363 (1991), 185

[5] G. Amelino-Camelia, I.I. Kogan and R.J. Szabo, Intern. J. Mod. Phys. A12 (1997), 1043

[6] I.I. Kogan, Phys. Lett. B256 (1991), 369

[7] S. Carlip, Nucl. Phys. B362 (1991), 111

[8] I.I. Kogan, Nucl. Phys. B375 (1992), 362;

M.C. Ashworth, Mod. Phys. Lett. A10 (1995), 2749

[9] E. Witten, Commun. Math. Phys. 121 (1989), 351;

G. Moore and N. Seiberg, Phys. Lett. B220 (1989), 220;

S. Elitzur, G. Moore, A. Schwimmer and N. Seiberg, Nucl. Phys. B326 (1989), 108;

M. Bos and V.P. Nair, Phys. Lett. B223 (1989), 61; Intern. J. Mod. Phys. A5 (1990), 959

W. Ogura, Phys. Lett. B229 (1989), 61;

N. Sakai and Y. Tanii, Progr. Theor. Phys. 83 (1990), 968

[10] I.I. Kogan, Phys. Lett. B231 (1989), 377

[11] S. Carlip and I.I. Kogan, Phys. Rev. Lett. 64 (1990), 148; Mod. Phys. Lett. A6 (1991), 171

[12] G. Amelino-Camelia, I.I. Kogan and R.J. Szabo, Nucl. Phys. B480 (1996), 413

[13] Y. Aharonov and D. Bohm, Phys. Rev. 115 (1959), 485

[14] A.M. Polyakov, Mod. Phys. Lett A2 (1987), 893;

V.G. Knizhnik, A.M. Polyakov and A.B. Zamolodchikov, Mod. Phys. Lett. A3 (1988), 819 ;

F. David, Mod. Phys. Lett. A3 (1988), 1651;

J. Distler and H. Kawai, Nucl. Phys. B321 (1989), 509

[15] S. Carlip and I.I. Kogan, Phys. Rev. Lett. 67 (1991), 3647

[16] S. Deser and X. Xiang, Phys. Lett. B263 (1991), 39

[17] A. Achucaro and P.K. Townsend, Phys. Lett. B180 (1986), 89;

E. Witten, Nucl. Phys. B311 (1988), 46; B323 (1989), 113;

S. Carlip, Nucl. Phys. B324 (1989), 106

[18] M.I. Dobroliubov, D. Eliezer, I.I. Kogan, G.W. Semenoff and R.J. Szabo, Mod. Phys. Lett. A8 (1993), 2177

[19] I.I. Kogan, Phys. Lett. B262 (1991), 83;

I.I. Kogan and G.W. Semenoff, Nucl. Phys. B368 (1992), 718;

R.J. Szabo, I.I. Kogan and G.W. Semenoff, Nucl. Phys. B392 (1993), 700 
[20] I.R. Klebanov, Phys. Rev. D51 (1995), 1836

[21] A. Staruszkiewicz, Acta Phys. Pol. 24 (1963), 734;

S. Deser, R. Jackiw and G. 't Hooft, Ann. Phys. 152 (1984), 220

[22] P.O. Mazur, Phys. Rev. Lett. 57 (1986), 929; 59 (1987), 2380; Phys. Rev. D34 (1986), 1925

[23] K. Ait Moussa and G. Clément, Class. Quant. Grav. 13 (1996), 2319

[24] J.-H. Cho and H.-J. Lee, Phys. Lett. B351 (1995), 111 